\title{
Patterns, Memory and Periodicity in Two-Neuron Delayed Recurrent Inhibitory Loops
}

\author{
J. $\mathbf{M a}^{1}$ and $\mathbf{J} . \mathbf{W u}^{2} *$ \\ ${ }^{1}$ Department of Mathematics, University of Houston, Houston TX 77204-3008, USA \\ ${ }^{2}$ Center for Disease Modeling; Laboratory for Industrial and Applied Mathematics, \\ Department of Mathematics and Statistics, York University, Toronto, Ontario, M3J 1P3, Canada
}

\begin{abstract}
We study the coexistence of multiple periodic solutions for an analogue of the integrateand-fire neuron model of two-neuron recurrent inhibitory loops with delayed feedback, which incorporates the firing process and absolute refractory period. Upon receiving an excitatory signal from the excitatory neuron, the inhibitory neuron emits a spike with a pattern-related delay, in addition to the synaptic delay. We present a theoretical framework to view the inhibitory signal from the inhibitory neuron as a self-feedback of the excitatory neuron with this additional delay. Our analysis shows that the inhibitory feedbacks with firing and the absolute refractory period can generate four basic types of oscillations, and the complicated interaction among these basic oscillations leads to a large class of periodic patterns and the occurrence of multistability in the recurrent inhibitory loop. We also introduce the average time of convergence to a periodic pattern to determine which periodic patterns have the potential to be used for neural information transmission and cognition processing in the nervous system.
\end{abstract}

Key words: multistability, periodic pattern, neural network, time delay, pattern formation, recurrent inhibitory loops

AMS subject classification: 34K13, 34K18, 92B20

${ }^{*}$ Corresponding author. E-mail: wujh@mathstat.yorku.ca 


\section{Introduction}

In a living nervous system, recurrent loops involving two or more neurons are ubiquitous and are particularly prevalent in cortical regions for memory such as the hippocampal-mesial temporal lobe complex [26]. The recurrent neural loops include the multiple pathways involved in the control of movement [2], reciprocal thalamocortical loops involved in epileptic seizures [11] and regulation of states of arousal [6], the limbic nervous system loops related to memory [1] and epileptic seizures [24], and the cortical-basal ganglia-thalamus-brain stem-cortical loops, which participate in the control of movement and act as gate keepers for the propagation of epileptic seizures [23].

In this paper, we consider a simple recurrent inhibitory loop consisting of an excitatory neuron $E$ and an inhibitory neuron $I$, where neuron $E$ gives off collateral branches and excites the inhibitory neuron $I$, which in turns inhibits the firing of neuron $E$, in a delay time. Such twoneuron recurrent inhibitory loops with delay display similar complex dynamic behaviors as larger networks and many techniques developed to deal with two-neuron networks can carry over to networks of large size. Moreover, two-neuron networks are sometimes thought of as systems of two modules, where each module represents the mean activity of a spatially localized neural population $[3,25]$.

The focus of this paper is on the capacity of the recurrent inhibitory loop to generate multiple coexisting periodic patterns (multistability). The coexistence of multiple stable patterns in neural networks is the basis of the mechanism for (associative) content-addressable memory storage and retrieval $[8,13,14,20,22]$ where each stable equilibrium is identified with a static memory, while stable periodic orbits are associated with temporally patterned spike trains $[4,8,9]$. Periodic patterns exhibited in neural networks have been linked to a variety of rhythms, which are associated with important behavioral and cognitive states in the nervous system, including attention, working memory, associative memory, object recognition, sensory motor integration and perception processing $[5,7,15]$.

Time delays, a powerful mechanism for multistability, are intrinsic properties of the nervous systems and are unavoidable in electronic implementation due to axonal conduction times, distances of interneurons and the finite switching speeds of amplifiers. Multistability in a delayed neural network has been extensively studied in the literature, in particular for delayed neural recurrent loops [8,9], and experimentally in electrical circuits [9] and in recurrently clamped neurons [10]. Foss et al. [8] studied neural recurrent inhibitory loops using the well-known HodgkinHuxley model and found three coexisting attracting periodic solutions by computer simulation. $\mathrm{Ma}$ and $\mathrm{Wu}[17,18]$ showed that the phenomenological spiking neuron model incorporating the firing process and the absolute refractory period can generate a large number of asymptotically stable periodic solutions with predictable patterns of oscillations. However, the aforementioned work $[8,17,18]$ on inhibitory loops/feedbacks is based on the consideration of the dynamical behaviors of the single excitatory neuron in the two-neuron inhibitory loop by simplifying the effect of the inhibitory neuron on the excitatory neuron as an inhibitory self-feedback in the recurrent loops.

In this paper, we study both the excitatory and inhibitory neurons and consider a system of coupled delay differential equations. We develop a systematical approach to rigorously analyze the mechanism for the coexistence of multiple periodic patterns in the recurrent inhibitory loop. 
In particular, the interaction among the excitatory and inhibitory neurons, time lag, firing process and the absolute refractory period can generate some basic components of periodic patterns. This allows us to link the solution semiflow defined by the model to symbolic dynamics in which these basic components can be pinned together to form a large class of periodic patterns and the model exhibits rich dynamics in the form of the coexisting periodic patterns. Moveover, an important quantity, the average time of convergence to a periodic pattern, can help us to determine what kind of patterns can be potentially used for neural information transmission, object recognition, sensory and perception processing in the nervous system.

The rest of this paper is organized as follows: we first formulate the two-dimensional integrateand-fire neuron model for the recurrent inhibitory loop which incorporates the firing process and the absolute refractory period in Section 2. In Section 3, we show that the interaction of time lag, inhibitory feedback, firing and absolute refractory period can generate four types of basic oscillations $\left(V, W_{u}, W_{d}\right.$ and $W_{c}$ ) for the excitatory neuron, and we describe in details the corresponding basic types of the oscillations for the inhibitory neuron. Section 4 is devoted to developing a theoretical framework to view the inhibitory feedback from the inhibitory neuron as a self-feedback of the excitatory neuron: we note that the inhibitory neuron, upon receiving an excitatory signal from the excitatory neuron, emits a spike with a delay. This delay is in addition to the synaptic delay. The size of this additional delay is associated with the specific type of oscillation of the inhibitory neuron, and is determined fully by the period of the corresponding type of oscillation of the excitatory neuron. In summary, we show that the inhibitory signal from the inhibitory neuron can be regarded as a self-feedback of the excitatory neuron with this additional delay. In Section 5 , we develop general principles that determine how these building blocks of oscillations interact each other to generate multiple periodic patterns, and apply these general principles to an example in Section 6 to illustrate the periodic patterns we theoretically derive. Section 7 provides some numerical simulations to illustrate similar periodic patterns exhibited by the more realistic model of the recurrent loop with an alpha synaptic function. Some remarks and discussions are presented in the final section.

\section{Model of Recurrent Inhibitory Loops and Simulation Re- sults}

We consider the following normalized integrate-and-fire model of a recurrent inhibitory loop:

$$
\left\{\begin{array}{l}
V_{E}^{\prime}(t)=-V_{E}(t)-F_{I}(t)+I_{0} \\
V_{I}^{\prime}(t)=-V_{I}(t)+F_{E}(t, \tau)
\end{array}\right.
$$

where $I_{0}$ is the external input (assumed to be a constant), $F_{E}(t, \tau)$ describes the excitatory input from the excitatory neuron $E$ to the inhibitory neuron $I$ and $F_{I}(t)$ describes the inhibitory input from neuron $I$ to neuron $E$. The dynamical behaviors of both excitatory and inhibitory neurons are subject to a resetting mechanism. Namely, once these potentials reach the firing threshold $\vartheta$ at a firing time $t_{f}$, there is a spike followed by an absolute refractory period $\left(t_{f}, t_{f}+T_{F R}\right)$ during which both excitatory and inhibitory neurons are unresponsive to any input, and their potentials are 
reset to the after-potential $V_{A}$. Here $T_{F R}$ is the sum of the firing process and the absolute refractory period. The firing time $t_{f}$ is defined by the threshold condition:

$$
V_{i}\left(t_{f}\right)=\vartheta \quad \text { and } \quad V_{i}\left(t_{f}-\varepsilon\right)<\vartheta, \quad \text { where } \mathrm{i}=(\mathrm{E}, \mathrm{I})
$$

for sufficiently small $\varepsilon>0$.

Once neuron $E$ fires, an excitatory postsynaptic potential (EPSP) is delivered to neuron $I$ with a time lag $\tau$. The excitatory input is given by

$$
F_{E}(t, \tau)= \begin{cases}b & \text { if } t \in\left[t_{f, E}^{\tau}+\tau, t_{f, E}^{\tau}+\tau+T_{E F}\right] \\ 0 & \text { otherwise }\end{cases}
$$

where $T_{E F}$ is the duration of the excitatory input and $t_{f, E}^{\tau}$ is the last firing time of neuron $E$ prior to the time $t-\tau$. More precisely, $t_{f, E}^{\tau}=\sup \left\{s ; s \leq t-\tau, V_{E}(s)=\vartheta\right.$ and $V(s-\varepsilon)<\vartheta$ for sufficiently small $\varepsilon>0\}$. We assume that the excitatory input $F_{E}(t, \tau)$ will cause neuron $I$ to fire and trigger only one spike. Hence, we take $b>\vartheta$. In order to simplify our analysis, we assume $T_{E F}$ is set appropriately so that once neuron $I$ emits a spike, there will be no further impact of the EPSP on neuron $I$ (If $T_{E F}$ is greater than such a value, the computation is slightly more complicated, but it does not change our final results).

In turn, neuron $I$ instantaneously delivers an inhibitory postsynaptic potential (IPSP) to inhibit the firing of neuron $E$. The inhibitory input is given by

$$
F_{I}(t)= \begin{cases}a & \text { if } t \in\left[t_{f, I}, t_{f, I}+T_{I F}\right] \\ 0 & \text { otherwise }\end{cases}
$$

where $T_{I F}$ is the duration of the inhibitory input and $t_{f, I}$ is the firing time of neuron $I$. It is reasonable to assume that $T_{F R}<T_{I F}<T$, where $T$ is the intrinsic period of neuron $I$, to be defined in Subsection 3.1. We also assume that $a>I_{0}-V_{A}$ so that the inhibitory input will cause the potential of neuron $E$ to decline. However, if the inhibitory input is delivered during the firing period or the absolute refractory period of neuron $E$, the input has no effect on the membrane potential during these two periods.

Note that we assume that neuron $I$ delivers an IPSP to neuron $E$ instantaneously (i.e. without a synaptic delay). Such a synaptic delay can be easily incorporated into the recurrent loop model, but we can also omit this synaptic delay by a simple change of variables.

\subsection{Simulation Results}

To illustrate basic oscillations and the formation of periodic patterns exhibited by neuron $E$ in the recurrent inhibitory loop, we briefly present some simulation results. The firing process is represented by a piecewise linear function (the membrane potential first increases from the firing threshold $\vartheta$ to the amplitude $c$ of a spike, then decays to a reset potential $V_{r}$ ), and the followed absolute refractory period is represented by an exponential function (the potential to increase $V_{r}$ to the after-potential $V_{A}$ ). To obtain all possible periodic patterns, we have performed experiments using a variety of initial conditions. 

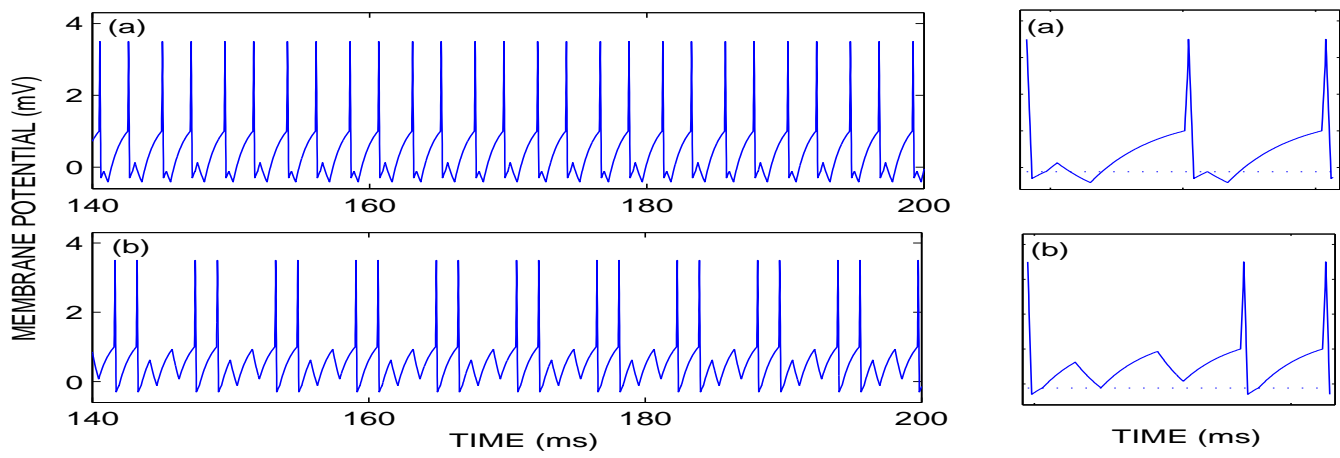

Figure 1: Two coexisting attracting periodic solutions for $\tau=2.406 \mathrm{~ms}$ when $\vartheta=1 \mathrm{mV}$ : (a) $\left(W_{u} W_{d}\right)$ and (b) $\left(W_{u u} V\right)$. Other parameters are $I_{0}=1.45 \mu \mathrm{A}, a=2.7 \mu \mathrm{A}, b=6 \mu \mathrm{A}, T_{I F}=0.5 \mathrm{~ms}$, $T_{F R}=0.35 \mathrm{~ms}, V_{r}=-0.3 \mathrm{mV}, V_{A}=-0.1187 \mathrm{mV}$ and amplitude of spikes is $3.5 \mathrm{mV}$. The righthand side is the blow-up of solutions in a given period (not delay $\tau$ ) to clearly illustrate the patterns of solutions (the dotted line represents the after-potential $V_{A}$.
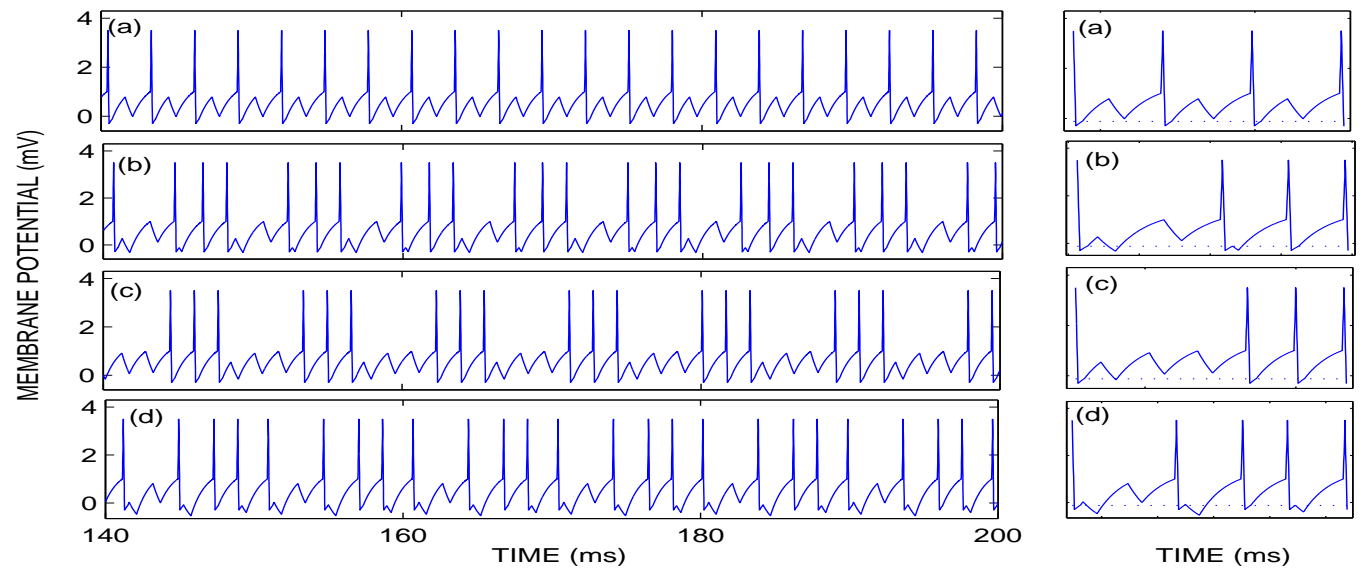

Figure 2: Four coexisting attracting periodic solutions when $\tau=3.917 \mathrm{~ms}$ and other parameters identical to those used in Figure 1: (a) $\left(W_{u}\right)$, (b) $\left(W_{u u} W_{d} V\right)$, (c) $\left(W_{u u u} 2 V\right)$ and (d) $\left(W_{u u} W_{u} V W_{u}\right)$. The dotted line in the right side represents the after-potential $\left.V_{A}\right)$.

Figure 1 shows two coexisting attracting periodic solutions when $\tau=2.406 \mathrm{~ms}, \vartheta=1 \mathrm{mV}$, $I_{0}=1.45 \mu \mathrm{A}, a=2.7 \mu \mathrm{A}, b=6 \mu \mathrm{A}, T_{I F}=0.5 \mathrm{~ms}, T_{F R}=0.35 \mathrm{~ms}, V_{r}=-0.3 \mathrm{mV}$ and $V_{A}=-0.1187 \mathrm{mV}$. The right-hand side is the blow-up of solutions in a given period (not delay $\tau$ ) to clearly illustrate the corresponding patterns. Focusing on the downstream oscillatory part of action potentials of these patterns, we note four types of basic oscillations: two types of $W$-oscillations as shown in Figure 1 (a), denoted by $W_{u}$-oscillation and $W_{d}$-oscillation, a more complicated oscillation, denoted by $W_{u u}$ and a simple oscillation, denoted by $V$-oscillation in Figure 1 (b). Intuitively, $u$ stands for "up" indicating that the membrane potential increases before 
an inhibitory signal is delivered; $d$ stands for "down" indicating that the membrane potential decreases due to the effect of an inhibitory signal; $V$ is a natural pattern where the action potential is not affected by an inhibitory signal. These periodic patterns in Figure 1 can be expressed in the order of these oscillations: (a) $\left(W_{u} W_{d}\right)$ and (b) $\left(W_{u u} V\right)$. Figure 2 shows four coexisting periodic patterns when $\tau=3.917 \mathrm{~ms}$ and other parameters are identical to those used in Figure 1: (a) $\left(W_{u}\right)$, (b) $\left(W_{u u} W_{d} V\right)$, (c) $\left(W_{u u u} 2 V\right)$ and (d) $\left(W_{u u} W_{u} V W_{u}\right)$. In the following sections, we shall define these basic oscillations and derive general principles of how periodic pattern formations develop.

\section{Basic Oscillations}

In this section, we define basic oscillations of neuron $E$ observed in our simulation, discuss the mechanisms for generating these oscillations and describe their implication for the oscillations exhibited by neuron $I$.

\subsection{The Excitatory Neuron}

In the absence of recurrent inhibition $\left(F_{I} \equiv 0\right)$, a strong enough stimulus $I_{0}\left(I_{0}>\vartheta\right)$ will cause neuron $E$ to emit a sequence of spikes, called a spike train. The period of such a spike train subject to firing and the absolute refractory period is called the intrinsic spiking period of neuron $E$, denoted by $T$. This period is the duration of two consecutive firing times and can be divided into two parts, $T_{F R}$ and $T_{A \vartheta}$,

$$
T=T_{F R}+T_{A \vartheta}, \quad T_{A \vartheta}=\log \left[\frac{I_{0}-V_{A}}{I_{0}-\vartheta}\right],
$$

where $T_{A \vartheta}$ is the time that the membrane potential of neuron $E$ increases from the after-potential $V_{A}$ to the threshold $\vartheta$.

Then we consider the impact of recurrent inhibitory input $F_{I}(t)$ on the dynamical behavior of neuron $E$. We note that each time neuron $I$ fires a spike, an inhibitory input instantaneously acts on neuron $E$. Both the number of delivered inhibitory signals and the arrival time of these signals in the cycle of an action potential (the duration between two consecutive firing times), play an important role in formatting an oscillation. We distinguish the number of the inhibitory signals delivered between two consecutive firing times in three cases: no inhibitory signal, only one signal and multiple signals, which will generate a $V$-oscillation, a $W_{d^{-}}$oscillation or $W_{u}$-oscillation, a $W_{c^{-}}$ oscillation, respectively. We use Figure 3 (a) to illustrate these basic oscillations $\left(V, W_{d}, W_{u}, W_{c}\right)$ corresponding to three cases.

\section{No Inhibitory Signal}

If there is no inhibitory signal delivered between two consecutive firing times, neuron $E$ emits a natural spike, called a $V$-oscillation, illustrated by the graph from $\mathrm{A}$ to $\mathrm{C}$ in Figure 3 (a). The period of a $V$-oscillation is equal to the intrinsic spiking period $T$ of neuron $E$, where $T_{F R}$ is the interval between $A$ and $B, T_{A \vartheta}$ is the interval between $B$ and $C$ in Figure 3 (a).

\section{Only One Inhibitory Input}



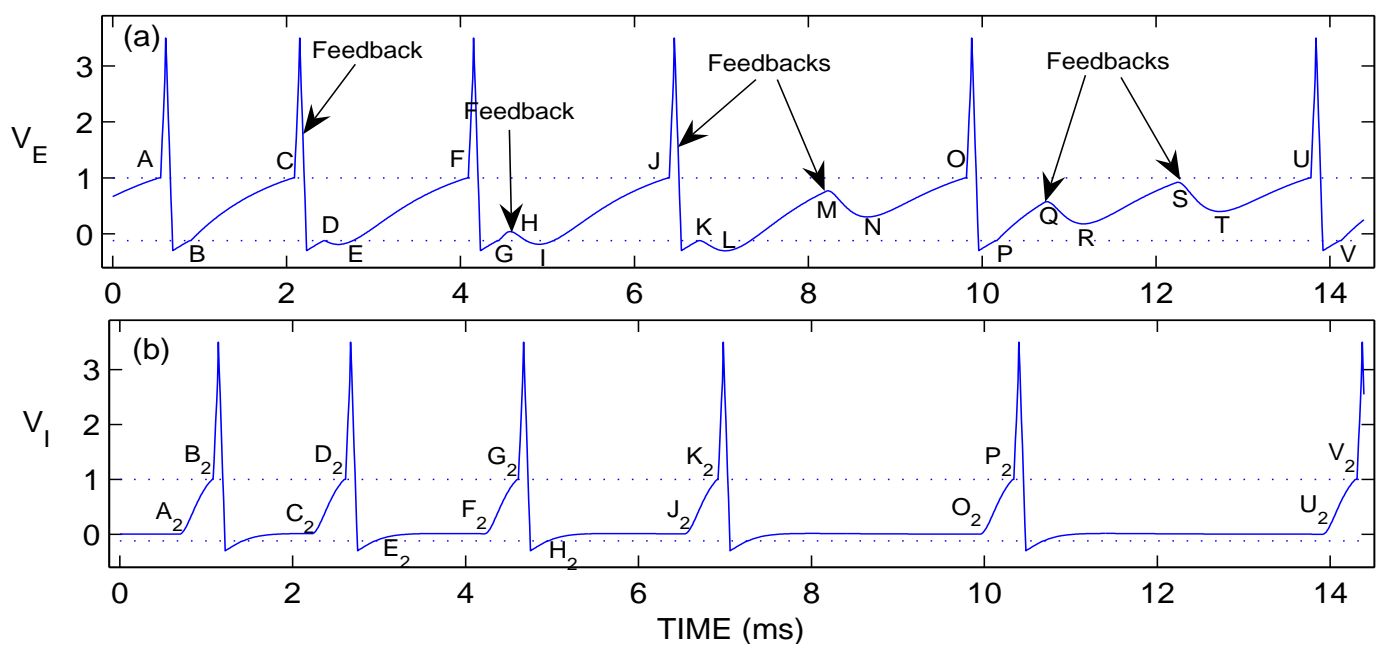

Figure 3: (a) Basic types of oscillations of the excitatory neuron and (b) the corresponding fundamental oscillations of the inhibitory neuron. In Figure 3 (a), from $A$ to $C$ is a $V$-oscillation; from $C$ to $F$ is a $W_{d}$-oscillation; from $F$ to $J$ is a $W_{u}$-oscillation; from $J$ to $O$ is a $W_{d u}$-oscillation; from $O$ to $U$ is a $W_{u u}$-oscillation. Each time the excitatory neuron $E$ fires a spike, an excitatory signal (EFB) is delivered to the inhibitory neuron $I$ in a time lag $\tau$. In Figure $3(\mathrm{~b})$, the excitatory signals delivered at $A_{2}, C_{2}, F_{2}, J_{2}, O_{2}$ and $U_{2}$ correspond to the spikes at $A, C, F, J, O$ and $U$ in (a), respectively. In order to associate with each spike, we moved the position of $V_{I}$ backwards by an amount of time delay $\tau$. The top dotted line indicates the threshold $\vartheta$ and the bottom line indicates the after-potential $V_{A}$.

If there is only one inhibitory input delivered between two firing times, and if this inhibitory signal is delivered during the firing process or the absolute refractory period (between $C$ and $D$ in Figure $3(\mathrm{a})$ ), since $T_{I F}>T_{F R}$, this inhibitory signal partially impacts on the membrane potential: after the absolute refractory period, the membrane potential first decreases in the effective inhibitory time (from $D$ to $E$ ), denoted by $t_{\text {down }}(\mathrm{ms})$, and then increases until the neuron fires. We call such an oscillation a $W_{d}$-oscillation, depicted from $C$ to $F$ in Figure 3 (a) ("d" stands for "down").

On the other hand, if this inhibitory signal is delivered after the absolute refractory period (at $H$ in Figure 3 (a)), this signal fully impacts on the membrane potential: after the absolute refractory period, the membrane potential first increases in a certain amount of time before the signal arrives, denoted by $t_{u p}$ (ms) (from $G$ to $H$ ), then decreases due to the effect of the inhibitory signal and finally increases until the neuron fires. We call such an oscillation a $W_{u}$-oscillation, depicted from $F$ to $J$ in Figure 3 (a) ("u" stands for "up").

The first quantity characterizing these two oscillations $\left(W_{d}\right.$ and $\left.W_{u}\right)$ is the effective inhibitory time, $t_{d o w n}$, for a $W_{d^{-}}$oscillation and the rising time, $t_{u p}$, prior to arrival of the signal for a $W_{u^{-}}$ oscillation. The second quantity to describe the inhibition of the signal is a time shift to the original cycle of the action potential. We denote by $t_{A \vartheta}$ the duration from the time when the inhibition of 
the signal wears off to the next firing time, depicted by the time from $E$ to $F$ for the $W_{d}$-oscillation and from $I$ to $J$ for the $W_{u}$-oscillation in Figure 3 (a). The time shift is $t_{d o w n}+t_{A \vartheta}-T_{A \vartheta}$ for a $W_{d}$-oscillation and $t_{u p}+T_{I F}+t_{A \vartheta}-T_{A \vartheta}$ for a $W_{u}$-oscillation. To simplify the notation, we denote $t_{A \vartheta}-T_{A \vartheta}$ by $\Delta_{d}$ or $\Delta t_{u}$ corresponding to a $W_{d}$ or $W_{u}$ oscillation. Then each $W_{d}$-oscillation can be characterized by a pair of variables $\left(t_{\text {down }}, \Delta t_{d}\right)$ with $T_{I F}-T_{F R}<t_{\text {down }} \leq T_{I F}$ and $\Delta t_{d}>0$, and its period is $T_{d}=T+t_{\text {down }}+\Delta t_{d}$. Each $W_{u}$-oscillation can be characterized by a triple of variables $\left(t_{u p}, T_{I F}, \Delta t_{u}\right)$ with $0<t_{u p}<T_{A \vartheta}$, and its period is $T_{u}=T+t_{u p}+T_{I F}+\Delta t_{u}$. The sign of $\Delta t_{u}$ depends on the value $t_{u p}$ : if the signal inhibition brings the membrane potential below $V_{A}, \Delta t_{u}>0$; otherwise, $\Delta t<0$.

\section{Multiple Inhibitory Signals}

There can be multiple inhibitory signals delivered between two consecutive firing times, which

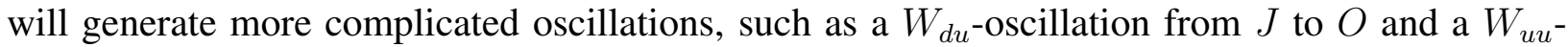
oscillation from $O$ to $U$ in Figure 3 (a). Similarly to $W_{d}$ and $W_{u}$ notations, the arrival time of the first signal determines either a $d$-stream characterized by $t_{d o w n}^{c}$ or a $u$-stream characterized by $\left(t_{u p}^{c}, T_{I F}\right)$. The followed signals are all of $u$-streams characterized by $\left(t_{u p}^{c}, T_{I F}\right)$. In Figure $3(\mathrm{a})$, the $W_{d u}$-oscillation results from two signals: the first signal delivered between $J$ and $K$ decreases the potential in an effective inhibitory duration $t_{\text {down }}^{c}$ (from $K$ to $L$ ) and the second signal is delivered at $M$ characterized by $\left(t_{u p}, T_{I F}\right)$, corresponding to the segments from $L$ to $M$ and from $M$ to $N$. The $W_{u u}$-oscillation is the effect of two signals delivered at $Q$ and $S$, characterized by $\left(t_{u p}^{c, 1}, T_{I F}\right)$ and $\left(t_{u p}^{c, 2}, T_{I F}\right)$, corresponding to each segment between $P$ and $T$. We call this type of oscillation a $W_{c}$-oscillation or $W_{c}^{m}$-oscillation ("c" for "complicated oscillation") with $c=d u \ldots u$ or $c=u \ldots u$, where $m$ is the total number of $d$ and $u$ 's in $c$ and it is the number of inhibitory signals. In general, a $W_{d u \ldots u}^{m}$-oscillation can be characterized by $\left(t_{d o w n}^{c}, t_{u p}^{c, 1}, T_{I F}, \ldots, t_{u p}^{c, m-1}, T_{I F}, \Delta t_{c}\right)$, where $T_{I F}-T_{F R}<t_{\text {down }}^{c} \leq T_{I F}$, and its period is $T+t_{\text {down }}^{c}+\sum_{i=1}^{m-1}\left(t_{u p}^{c, i}+T_{I F}\right)+\Delta t_{c} ;$ a $W_{u \ldots u}^{m}$-oscillation can be characterized by $\left(t_{u p}^{c, 1}, T_{I F}, \ldots, t_{u p}^{c, m}, T_{I F}, \Delta t_{c}\right)$ and its period is $T+\sum_{i=1}^{m}\left(t_{u p}^{c, i}+T_{I F}\right)+\Delta t_{c}$, where $\Delta t_{c}$ is defined in the same way as $\Delta_{d}$ and $\Delta_{u}$ described above.

\subsection{The Inhibitory Neuron}

Each time neuron $E$ fires a spike, an excitatory signal is delivered to neuron $I$ with a time lag $\tau$. In contrast to neuron $E$, we define a cycle of the membrane potential of neuron $I$ between two consecutive excitatory signals as a fundamental oscillation of neuron $I$. The excitatory signal delivered by a $V$-oscillation of neuron $E$ generates a fundamental oscillation of neuron $I$, denoted by $V^{I}$-oscillation, which is the graph from $A_{2}$ to $C_{2}$ in Figure 3 (b). Similarly, the spike of $W_{d^{-}}$ oscillation generates a fundamental $W_{d}^{I}$-oscillation (from $C_{2}$ to $F_{2}$ in Figure 3 (b)); the spike of $W_{u}$-oscillation generates a fundamental $W_{u}^{I}$-oscillation (from $F_{2}$ to $J_{2}$ in Figure 3 (b)); the spike of $W_{c}$-oscillation generates a fundamental $W_{c}^{I}$-oscillation, such as a $W_{d u}^{I}$-oscillation (from $J_{2}$ to $\mathrm{O}_{2}$ ) and a $W_{u u}^{I}$-oscillation (from $\mathrm{O}_{2}$ to $U_{2}$ ). It follows easily from the above discussion that the period of each fundamental oscillation of neuron $I$ is equal to the period of its corresponding basic oscillation of neuron $E$. 


\section{Pattern Formation From Four Basic Oscillations}

The interaction of inhibitory signals, the time lag, firing and the absolute refractory period can generate four basic oscillations $\left(V, W_{d}, W_{u}, W_{c}\right)$, which are the basic building blocks of periodic patterns exhibited by neuron $E$. In this section, we discuss the general principles of how these basic oscillations interact to generate a large class of periodic patterns of neuron $E$.

If neuron $E$ fires a spike at $t_{f}$, neuron $I$ receives an excitatory signal in a time lag $\tau$ due to such a spike, which excites neuron $I$ to fire. Once neuron $I$ emits a spike, an inhibitory signal is immediately delivered to neuron $E$. Such an inhibitory signal can be considered as a self-feedback (SFB) of neuron $E$ due to the spike at $t_{f}$.

For the inhibitory neuron, we denote the duration from the time that an excitatory signal arrives until neuron $I$ fires by $\tau_{A}$, which is the segment, $A_{2} B_{2}$, or $C_{2} D_{2}$, or $F_{2} G_{2}$, or $J_{2} K_{2}$, or $O_{2} P_{2}$, or $U_{2} V_{2}$ in Figure 3 (b). In contrast to the synaptic delay $\tau$, we call $\tau_{A}$ an additional delay. Hence, corresponding to each spike of the excitatory neuron, there is always an inhibitory self-feedback (SFB) to act on the excitatory neuron in a time delay $\tau^{\prime}=\tau+\tau_{A}$. We shall show that $\tau_{A}$ only depends on the aforementioned basic types of oscillations of the excitatory neuron when a periodic solution is generated. The advantage of introducing this additional delay is to help us to regard the IPSP as a self-feedback of the excitatory neuron so that we can focus on analyzing the dynamics of the excitatory neuron only.

For a given initial condition, the effect of such self-feedbacks eventually stabilizes the corresponding trajectory of neuron $E$ to a periodic solution with a predicted pattern composed of the aforementioned basic oscillations. Then a periodic pattern can be expressed by

$$
\sigma=\left(\pi_{1}, \pi_{2}, \ldots, \pi_{N-1}, \pi_{N}\right) \quad \text { where } \quad \pi_{i} \in\left\{V, W_{d}, W_{u}, W_{c}\right\} .
$$

We denote by $T_{i}:=T\left(\pi_{i}\right)$ the period of the basic oscillation $\pi_{i}$ and by $p:=\sum_{i=1}^{N} T_{i}$ the period of such a pattern. We note that a pattern which is the permutation of these oscillations $\pi_{1}, \ldots, \pi_{N}$ is equivalent to the above pattern, i.e., $\sigma_{1}=\left(\pi_{2}, \pi_{3}, \ldots, \pi_{N}, \pi_{1}\right)$ is equivalent to $\sigma$.

\subsection{The Inhibitory Neuron}

We denote the fundamental oscillations of the inhibitory neuron generated by spikes of $\pi_{1}, \pi_{2}, \ldots, \pi_{N}$ by $\pi_{1}^{I}, \pi_{2}^{I}, \ldots, \pi_{N}^{I}$, respectively. Additional delays corresponding to these fundamental oscillations $\pi_{1}^{I}, \pi_{2}^{I}, \ldots, \pi_{N}^{I}$ are denoted by $\tau_{1}, \tau_{2}, \ldots, \tau_{N}$, respectively. In what follows, we show that $\tau_{1}, \ldots, \tau_{N}$ can be determined by $T_{1}, \ldots, T_{N}$ via a single scalar function.

Theorem 1. For a given periodic pattern $\sigma=\left(\pi_{1}, \pi_{2}, \ldots, \pi_{N-1}, \pi_{N}\right)$ with $\pi_{i} \in\left\{V, W_{d}, W_{u}, W_{c}\right\}$, there exists a continuous function $g$ such that $\tau_{1}=g\left(T_{1}, T_{2}, \ldots, T_{N}\right), \tau_{2}=g\left(T_{2}, T_{3}, \ldots, T_{1}\right)$, ..., $\tau_{N}=g\left(T_{N}, T_{1}, \ldots, T_{N-1}\right)$.

Proof: We first illustrate our argument by a simple case where $\sigma=\left(\pi_{1}, \pi_{2}\right)$. We denote the membrane potential of the inhibitory neuron by $V_{1}^{I}$ at the time when the excitatory signal due to the spike $\pi_{1}$ is delivered, and by $V_{2}^{I}$ at the time when the excitatory signal due to the spike $\pi_{2}$ is delivered. 
Now we discuss the time course of membrane potential of the fundamental oscillation $\pi_{1}^{I}$. The potential evolves in three steps: (i) increasing from $V_{1}^{I}$ to the threshold; (ii) the firing process and the absolute refractory period; (iii) increasing from $V_{A}$ to $V_{2}^{I}$. We follow these steps to calculate $V_{1}^{I}$ and $V_{2}^{I}$.

First Step: Starting from the time when the excitatory signal due to the spike $\pi_{1}$ is delivered, it takes $\tau_{1}$ (ms) for the potential to increase from $V_{1}^{I}$ to the firing threshold $\vartheta$. Integrating the second equation in (2.1) yields

$$
\vartheta=V_{1}^{I} e^{-\tau_{1}}+b\left(1-e^{-\tau_{1}}\right) .
$$

Second Step: After neuron $I$ fires a spike and passes the absolute refractory period, the potential reaches the after-potential $V_{A}$.

Third Step: After firing and the absolute refractory period, it takes the duration of $T_{1}-\tau_{1}-T_{F R}$ for the potential to increase from the after-potential $V_{A}$ to $V_{2}^{I}$. System (2.1) gives rise to

$$
V_{2}^{I}=V_{A} e^{-\left(T_{1}-\tau_{1}-T_{F R}\right)}
$$

Similarly, we consider the fundamental oscillation $\pi_{2}^{I}$ and obtain two equations analogous to equations (4.2) and (4.3). Combining these equations together, we obtain

$$
\begin{array}{ll}
\vartheta=V_{1}^{I} e^{-\tau_{1}}+b\left(1-e^{-\tau_{1}}\right), & V_{1}^{I}=V_{A} e^{-\left(T_{2}-\tau_{2}-T_{F R}\right)}, \\
\vartheta=V_{2}^{I} e^{-\tau_{2}}+b\left(1-e^{-\tau_{2}}\right), & V_{2}^{I}=V_{A} e^{-\left(T_{1}-\tau_{1}-T_{F R}\right)} .
\end{array}
$$

We solve $\tau_{1}, \tau_{2}$ to obtain

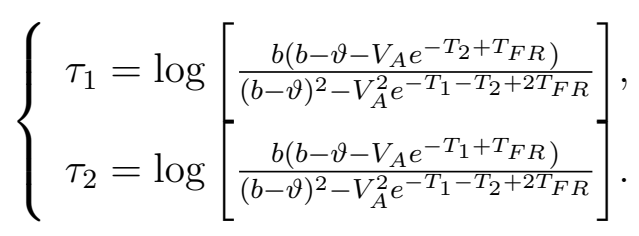

Therefore, the function $g$ is given by

$$
g\left(x_{1}, x_{2}\right)=\log \left[\frac{b\left(b-\vartheta-V_{A} e^{-x_{2}+T_{F R}}\right)}{(b-\vartheta)^{2}-V_{A}^{2} e^{-x_{1}-x_{2}+T_{F R}}}\right] .
$$

Clearly, $\tau_{1}=g\left(T_{1}, T_{2}\right)$ and $\tau_{2}=g\left(T_{2}, T_{1}\right)$.

Applying the same argument to the periodic pattern $\sigma=\left(\pi_{1}, \pi_{2}, \pi_{3}\right)$, we obtain

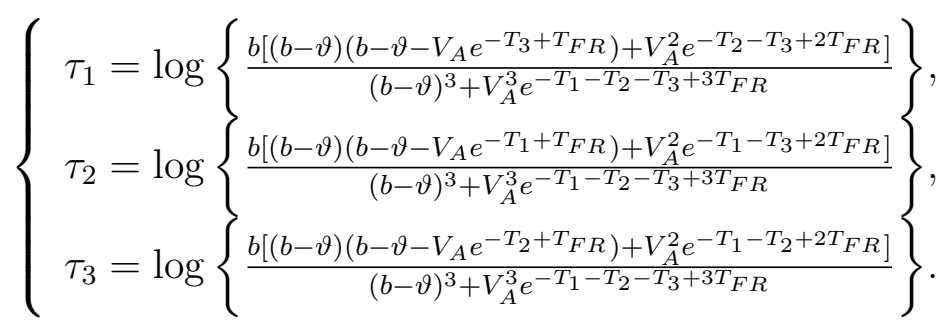


The function $g$ is given by

$$
g\left(x_{1}, x_{2}, x_{3}\right)=\log \left\{\frac{b\left[(b-\vartheta)\left(b-\vartheta-V_{A} e^{-x_{3}+T_{F R}}\right)+V_{A}^{2} e^{-x_{2}-x_{3}+2 T_{F R}}\right]}{(b-\vartheta)^{3}+V_{A}^{3} e^{-x_{1}-x_{2}-x_{3}+3 T_{F R}}}\right\}
$$

and $\tau_{1}=g\left(T_{1}, T_{2}, T_{3}\right), \tau_{2}=g\left(T_{2}, T_{3}, T_{1}\right)$ and $\tau_{3}=g\left(T_{3}, T_{1}, T_{2}\right)$.

This argument can apply for the general periodic pattern $\sigma=\left(\pi_{1}, \pi_{2}, \ldots, \pi_{N-1}, \pi_{N}\right)$. This completes our proof.

Therefore, there exists a single continuous function to describe the relationship of each $\tau_{i}$ and $T_{1}, \ldots, T_{N}$. The observed cyclic property can be interpreted as follows: if $\tau_{1}$ is considered as the function $g$ with respect to the periodic pattern $\sigma=\left(\pi_{1}, \pi_{2}, \ldots, \pi_{N}\right)$, then $\tau_{i}$ is the function $g$ with respect to the equivalent periodic pattern $\sigma_{i}=\left(\pi_{i}, \pi_{i+1}, \ldots, \pi_{i-1}\right)$. In other words, the additional delay $\tau_{i}$ can be expressed in terms of the function $g$ with respect to the equivalent periodic pattern.

In what follows, we use notations of $\tau_{v}, \tau_{u}, \tau_{d}, \tau_{c}$ (such as $\tau_{d u}, \tau_{u u}$ ) to denote the additional

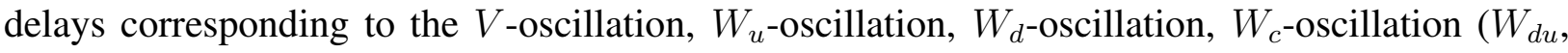
$\left.W_{u u}\right)$, respectively.

\subsection{The Excitatory Neuron}

After introducing the self-feedback (SFB) and establishing the relationship of $\tau_{1}, \ldots, \tau_{N}$ and $T_{1}, \ldots, T_{N}$, we can now concentrate on the excitatory neuron to study periodic patterns. We present the general principles to determine how the aforementioned basic oscillations are interacted to form a periodic pattern.

For a given periodic pattern $\sigma=\left(\pi_{1}, \pi_{2}, \ldots, \pi_{N-1}, \pi_{N}\right)$ where $\pi_{i} \in\left\{V, W_{d}, W_{u}, W_{c}\right\}$, we consider an action potential $\pi_{i}$ which delivers a self-feedback (SFB) to the action potential $\pi_{i+R}$ with $0 \leq R \leq N-1$. The action of the self-feedback (SFB) from $\pi_{i}$ on $\pi_{i+R}$ yields

$$
\begin{cases}\sum_{k=i}^{i+R-1} T_{k}+T_{F R} \geq \tau+\tau_{i}+T_{I F} \text { and } \pi_{i}=W_{c} & \text { iff } \pi_{i+R}=V ; \\ \sum_{k=i}^{i+R-1} T_{k}+T_{F R}+t_{\text {down }}=\tau+\tau_{i}+T_{I F} & \text { iff } \pi_{i+R}=W_{d} ; \\ \sum_{k=i}^{i+R-1} T_{k}+T_{F R}+t_{u p}=\tau+\tau_{i} & \text { iff } \pi_{i+R}=W_{u} ; \\ \cdot \sum_{k=j}^{i+R} T_{k}+T_{F R}+t_{d o w n}^{c}=\tau+\tau_{i}+T_{I F} & \text { iff } d \text { of } \pi_{i+R}=W_{d u \ldots u}^{m} ; \\ \sum_{k=i}^{i+R}-1 T_{k}+T_{F R}+t_{\text {down }}^{c}+\sum_{j=1}^{h}\left(t_{u p}^{c, j}+T_{F D}\right) & \\ =\tau+\tau_{i}+T_{I F} & \text { iff the } h \text {-th } u \text { of } \pi_{i+R}=W_{d u \ldots u}^{m} \\ \sum_{k=i}^{i+R-1} T_{k}+T_{F R}+\sum_{j=1}^{h} t_{u p}^{c, j}=\tau+\tau_{i} & \text { iff the } h \text {-th } u \text { of } \pi_{i+R}=W_{u \ldots u}^{m}\end{cases}
$$

where $\tau_{i}$ is the additional delay corresponding to the basic oscillation $\pi_{i}$, given by $\tau_{i}=g\left(T_{i}, T_{i+1} \ldots, T_{i-1}\right)$. Analogously, $N-1$ other patterns equivalent to $\sigma$ give $N-1$ relationships, which determine the types of periodic patterns generated by the recurrent inhibitory loop for a given time delay. Furthermore, these relationships determine the minimum and maximum values of $\tau$ for the existence of a given periodic pattern.

Now we consider the possible value of $R$ to determine all possible periodic patterns exhibited by the excitatory neuron. According to the value of $R$, we distinguish periodic patterns in four cases, as shown in Figure 4: 


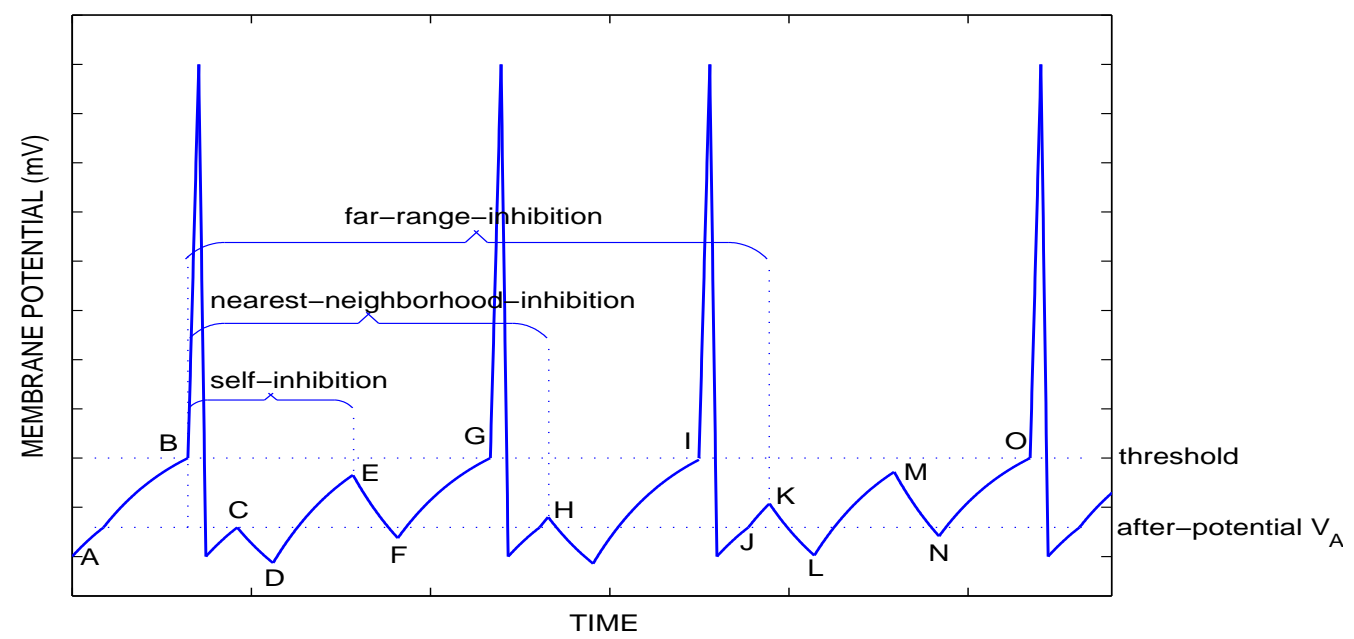

Figure 4: Schematic of three types of periodic patterns composed of a $W_{c}$-oscillation (from $B$ to $G$ ). When the excitatory neuron fires a spike at $B$, an inhibitory self-feedback is delivered at a time lag $\tau^{\prime}=\tau+\tau_{A}$. If this feedback is delivered to the $W_{c}$-oscillation itself (at E), it is called a self-inhibitory pattern; if the feedback is delivered to its nearest neighbor of $W_{c}$-oscillation (at $H$ ), it is called a nearest-neighbor-inhibitory pattern; otherwise, a far-range-inhibitory pattern is generated (for example, the feedback arrives at $K$ ).

- $R=N-1$ : the first item $\pi_{1}$ always acts on the last item $\pi_{N}$ within the pattern $\sigma$ and this periodic pattern is composed of only $W_{u}$ and $W_{d}$ oscillations;

- $R=0$ for $\pi_{i}=W_{c}$ : the spike of $W_{c}$-oscillation delivers a self-feedback to itself and we call it a self-inhibitory pattern;

- $R=1$ for $\pi_{j}=W_{c}$ : the spike of $W_{c}$-oscillation delivers a self-feedback to its nearest neighbor and we call it a nearest-neighbor-inhibitory pattern;

- $R>1$ for $\pi_{i}=W_{c}$ : the spike of $W_{c}$-oscillation delivers a self-feedback to an oscillation far away from its nearest neighbor and we call it a far-range-inhibitory pattern.

\section{Periodic Patterns Exhibited by the Excitatory Neuron}

In this section, we discuss four types of periodic patterns in details: periodic patterns composed of only $W_{u}$-oscillations and $W_{d}$-oscillations, self-inhibitory periodic patterns, nearest-neighborinhibitory periodic patterns and far-range-inhibitory patterns. 


\subsection{Periodic Patterns Composed of $W_{u}$-oscillations and $W_{d}$-oscillations}

If $R=N-1$ for all equivalent periodic patterns of $\sigma=\left(\pi_{1}, \pi_{2}, \ldots, \pi_{N}\right)$, then the first item $\pi_{1}$ always acts on the last item $\pi_{N}$ within the pattern $\sigma$. It follows easily from the definition in Subsection 3.1 that neither the $V$-oscillation nor the $W_{c}$-oscillation can appear in such a periodic pattern.

Lemma 2. For a given periodic pattern $\sigma=\left(\pi_{1}, \pi_{2}, \ldots, \pi_{N}\right)$ where each $\pi_{i}$ is one of the basic oscillation, if $R=N-1$ for all equivalent periodic patterns of $\sigma$, then $\pi_{i}$ is either a $W_{u}$-oscillation or a $W_{d^{-}}$oscillation.

Examples of periodic patterns composed of only $W_{u}$-oscillation and $W_{d}$-oscillation are $\left(W_{u} W_{d}\right)$ in Figure 1 (a) and $\left(1 W_{u}\right)$ in Figure 2 (a).

Lemma 3. For a given periodic pattern $\sigma=\left(\pi_{1}, \pi_{2}, \ldots, \pi_{N}\right)$ where $\pi_{i} \in\left\{W_{u}, W_{d}\right\}$, all $W_{u^{-}}$ oscillations must be the same in terms of $\left(t_{u p}, T_{I F}, \Delta t_{u}\right)$ and all $W_{d}$-oscillations must be the same in terms of $\left(t_{\text {down }}, \Delta t_{d}\right)$.

Proof: We illustrate our argument by a simple case, where $\sigma=\left(\pi_{1}, \pi_{2}\right)=\left(W_{u}^{(1)}, W_{u}^{(2)}\right) . \pi_{1}$ is characterized by $\left(t_{u p}^{(1)}, T_{I F}, \Delta t_{u}^{(1)}\right)$ and $\pi_{2}$ is characterized by $\left(t_{u p}^{(2)}, T_{I F}, \Delta t_{u}^{(2)}\right)$. Condition (4.6) gives rise to

$$
\left\{\begin{array}{l}
T_{2}+T_{F R}+t_{u p}^{(1)}=\tau+\tau_{2}, \\
T_{1}+T_{F R}+t_{u p}^{(2)}=\tau+\tau_{1} .
\end{array}\right.
$$

The above system yields

$$
T_{1}-t_{u p}^{(1)}-\tau_{1}=T_{2}-t_{u p}^{(2)}-\tau_{2}
$$

Substituting $T_{1}, T_{2}$ into the above equation, we obtain that $\Delta t_{u}^{(1)}-\tau_{1}=\Delta t_{u}^{(2)}-\tau_{2}$.

The potential of a $W_{u}$-oscillation evolves in three steps after the firing and the absolute refractory period: (i) increases from the after-potential $V_{A}$ to $V_{1}$ in the duration of $t_{u p}$ (ms); (ii) then decreases from $V_{1}$ to $V_{2}$ in the duration of $T_{I F}(\mathrm{~ms})$ due to an inhibitory feedback; (iii) after the feedback wears off, the potential increases from $V_{2}$ to the threshold $\vartheta$ in the duration of $t_{A \vartheta}=T_{A \vartheta}+\Delta t_{u}$. These three steps give rise to

$$
\begin{aligned}
& V_{1}=I_{0}-\left(I_{0}-V_{A}\right) e^{-t_{u p}}, \\
& V_{2}=I_{0}-a-\left(I_{0}-a-V_{1}\right) e^{-t_{u p}}, \\
& T_{A \vartheta}+\Delta t_{u}=\log \left[\frac{I_{0}-V_{2}}{I_{0}-\vartheta}\right] .
\end{aligned}
$$

Combining the above three equations and using equation (3.1) of $T_{A \vartheta}$, we obtain

$$
\left(I_{0}-V_{A}\right)\left[e^{\Delta t_{u}}-e^{-T_{I F}-t_{u p}}\right]=a\left(1-e^{-T_{I F}}\right) .
$$


For both the $W_{u}^{(1)}$-oscillation and $W_{u}^{(2)}$-oscillation, we have

$$
\begin{aligned}
& \left(I_{0}-V_{A}\right)\left[e^{\Delta t_{u}^{(1)}}-e^{-T_{I F}-t_{u p}^{(1)}}\right]=a\left(1-e^{-T_{I F}}\right), \\
& \left(I_{0}-V_{A}\right)\left[e^{\Delta t_{u}^{(2)}}-e^{-T_{I F}-t_{u p}^{(2)}}\right]=a\left(1-e^{-T_{I F}}\right) .
\end{aligned}
$$

Combining the above two equations with $\Delta t_{u}^{(1)}-\tau_{1}=\Delta t_{u}^{(2)}-\tau_{2}$, we obtain

$$
e^{\tau_{2}}\left(e^{-T_{F R}}-e^{-T_{1}}\right)=e^{\tau_{1}}\left(e^{-T_{F R}}-e^{-T_{2}}\right) .
$$

Substituting $\tau_{1}=g\left(T_{1}, T_{2}\right)$ and $\tau_{2}=g\left(T_{2}, T_{1}\right)$ of equation (4.4), we obtain

$$
\begin{aligned}
& {\left[\frac{b\left(b-\vartheta-V_{A} e^{-T_{1}+T_{F R}}\right)}{(b-\vartheta)^{2}-V_{A}^{2} e^{-T_{1}-T_{2}+2 T_{F R}}}\right]\left(e^{-T_{F R}}-e^{-T_{1}}\right)=\left[\frac{b\left(b-\vartheta-V_{A} e^{-T_{2}+T_{F R}}\right)}{(b-\vartheta)^{2}-V_{A}^{2} e^{-T_{1}-T_{2}+2 T_{F R}}}\right]} \\
& \times\left(e^{-T_{F R}}-e^{-T_{2}}\right) \text {. }
\end{aligned}
$$

It follows easily from the above equation that $T_{1}=T_{2}$. Hence, $t_{u p}^{(1)}=t_{u p}^{(2)}$ and $\Delta t_{u}^{(1)}=\Delta t_{u}^{(2)}$.

The same argument with $\tau_{i}=g\left(T_{i}, T_{i+1}, \ldots, T_{i-1}\right)$ can apply to show that all $W_{u}$-oscillations in a periodic pattern must be the same in terms of $\left(t_{u p}, T_{I F}, \Delta t_{u}\right)$ and all $W_{d}$-oscillations must be the same in terms of $\left(t_{\text {down }}, \Delta t_{d}\right)$.

Lemma 4. For a given periodic pattern $\sigma=\left(\pi_{1}, \pi_{2}, \ldots, \pi_{N}\right)$ where $\pi_{i} \in\left\{W_{u}, W_{d}\right\}$, all additional delays $\tau_{i}$ related to the $W_{u}$-oscillations must be the same and all additional delays $\tau_{i}$ related to the $W_{d}$-oscillations must be the same.

Lemma 5. For a given periodic pattern $\sigma=\left(\pi_{1}, \pi_{2}, \ldots, \pi_{N}\right)$ where $\pi_{i} \in\left\{W_{u}, W_{d}\right\}$, if a $W_{u^{-}}$ oscillation and $a W_{d}$-oscillation coexist in this periodic pattern, then $\Delta t_{u}-\tau_{u}=\Delta t_{d}-\tau_{d}$, where $\tau_{u}, \tau_{d}$ are the additional delays corresponding to the $W_{u}$-oscillation and the $W_{d^{-}}$oscillation, respectively.

Proof: Again, we illustrate our argument by a simple case where $\sigma=\left(\pi_{1}, \pi_{2}\right)=\left(W_{u}, W_{d}\right) . \pi_{1}$ is characterized by $\left(t_{u p}, T_{I F}, \Delta t_{u}\right)$ and $\pi_{2}$ is characterized by $\left(t_{d o w n}, \Delta t_{d}\right)$. Condition (4.6) gives rise to

$$
\left\{\begin{array}{l}
T_{1}+T_{F R}+t_{\text {down }}=\tau+\tau_{u}+T_{I F} \\
T_{2}+T_{F R}+t_{\text {up }}=\tau+\tau_{d}
\end{array}\right.
$$

The above system yields

$$
T_{1}-t_{u p}-\tau_{u}=T_{2}-t_{d o w n}+T_{I F}-\tau_{d}
$$

Substituting $T_{1}, T_{2}$ into the above equation, we obtain that $\Delta t_{u}-\tau_{u}=\Delta t_{d}-\tau_{d}$.

The relationship of $t_{d o w n}$ and $\Delta t_{d}$ for a $W_{d}$-oscillation is given by

$$
t_{\text {down }}=\log \left[\frac{I_{0}-V_{A}-a}{\left(I_{0}-V_{A}\right) e^{\Delta t_{d}}-a}\right] \text {. }
$$


The relationship of $t_{u p}$ and $\Delta t_{u}$ for a $W_{u}$-oscillation is given by

$$
t_{u p}=\log \left[\frac{I_{0}-V_{A}}{\left(I_{0}-V_{A}\right) e^{T_{I F}+\Delta t_{u}}-a e^{T_{I F}}+a}\right] .
$$

When $t_{\text {down }}=T_{I F}$ for a $W_{d}$-oscillation and $t_{u p}=0$ for a $W_{u}$-oscillation, the $W_{d}$-oscillation coincides with the $W_{u}$-oscillation $\left(\Delta t_{u}=\Delta t_{d}\right.$, and correspondingly $\left.\tau_{u}=\tau_{d}\right)$ and two action potentials are the same. In such a case, $\Delta t_{d}$ and $\Delta t_{u}$ reach their maximum values, denoted by

$$
\Delta t_{\text {max }}=\log \left[1+\frac{a\left(e^{T_{I F}}+1\right)}{I_{0}-V_{A}}\right]-T_{I F}>0 .
$$

For the $W_{d}$-oscillation, when $t_{d o w n}=T_{I F}-T_{F R}, \Delta t_{d}$ reaches its minimum value $\Delta t_{d}^{\text {min }}$ given by

$$
\Delta t_{d}^{\min }=\log \left[\frac{\left(I_{0}-V_{A}-a\right) e^{-\left(T_{I F}-T_{F R}\right)}+a}{I_{0}-V_{A}}\right]>0 .
$$

Theorem 6. The periodic pattern $\left(1 W_{u}\right)$ for $\sigma=\left(k W_{u}\right)$ with $k \geq 2$ can be generated if and only if

$$
\left\{\begin{array}{l}
t_{u p}=\frac{\tau+\tau_{u}-\left[(k-1) T+T_{F R}+(k-1) T_{I F}+(k-1) \Delta t_{u}\right]}{k}, \\
0<t_{u p}<T_{A \vartheta},
\end{array}\right.
$$

where $\tau_{u}$ is the additional delay of the $W_{u}$-oscillation. The minimum and maximum values of $\tau$ for the existence of such a periodic pattern are given by

$$
\left\{\begin{array}{l}
\tau_{\min }=(k-1) T+T_{F R}+(k-1)\left(T_{I F}+\Delta t_{\max }\right)-\left.\tau_{u}\right|_{\Delta t_{\max }}, \\
\tau_{\max }=(k-1) T+T_{F R}+(k-1) T_{I F}+k T_{A \vartheta}+(k-1) \Delta t_{u}^{\min }-\left.\tau_{u}\right|_{\Delta t_{u}^{\min }},
\end{array}\right.
$$

where $\left.\tau_{u}\right|_{\Delta t_{\max }}$ is the value of $\tau_{u}$ subject to the condition where $t_{u p}=0$ and $\Delta t_{u}=\Delta t_{\text {max }}$, and $\left.\tau_{u}\right|_{\Delta t_{u}^{\min }}$ is the value of $\tau_{u}$ subject to the condition where $t_{u p}=T_{A \vartheta}$ and $\Delta t_{u}=\Delta t_{u}^{\text {min }}$. Here

$$
\Delta t_{u}^{\min }=\log \left[\frac{I_{0}-\vartheta+a\left(e^{T_{I F}}-1\right)}{I_{0}-V_{A}}\right]-T_{I F}<0 .
$$

Proof: We consider $\sigma=\left(\pi_{1}, \ldots, \pi_{k}\right)=\left(W_{u}, \ldots, W_{u}\right)$ with the $W_{u}$-oscillation being characterized by $\left(t_{u p}, T_{I F}, \Delta t_{u}\right)$. Condition (4.6) gives rise to

$$
(k-1) T+(k-1)\left(t_{u p}+T_{I F}+\Delta t_{u}\right)+T_{F R}+t_{u p}=\tau+\tau_{u} .
$$

Re-arranging the above formula yields equation (5.5) and $t_{u p}$ must satisfy $0<t_{u p}<T_{A \vartheta}$.

When $t_{u p}=0, \Delta t_{u}$ reaches its maximum value $\Delta t_{\max }$ given by equation (5.3). The corresponding additional delay $\tau_{u}$ is denoted by $\left.\tau_{u}\right|_{\Delta t_{\max }}$. This situation gives rise to the minimum value $\tau_{\min }$ of equation (5.5).

On the other hand, as $t_{u p}$ approaches $T_{A \vartheta}$, equation (5.2) yields the value $\Delta t_{u}^{\text {min }}$ of equation (5.7). The corresponding additional delay $\tau_{u}$ is denoted by $\left.\tau_{u}\right|_{\Delta t_{u}^{m i n}}$. This situation yields the maximum value $\tau_{\max }$ of equation (5.5). 
Now we apply the above theorem to the simple pattern $\sigma=\left(2 W_{u}\right)$, as shown in Figure 2 (a). The period of a $W_{u}$-oscillation, condition (4.6), the additional time delay (4.4), and equation (5.2) yield

$$
\begin{aligned}
& T_{u}=T+t_{u p}+T_{I F}+\Delta t_{u}, \\
& T_{u}+T_{F R}+t_{u p}=\tau+\tau_{u}, \\
& \tau_{u}=\log \left[\frac{b\left(b-\vartheta-V_{A} e^{\left.-T_{u}+T_{F R}\right)}\right.}{(b-\vartheta)^{2}-V_{A}^{2} e^{-2 T_{u}+2 T_{F R}}}\right] \\
& t_{u p}=\log \left[\frac{I_{0}-V_{A}}{\left(I_{0}-V_{A}\right) e^{T_{I F}+\Delta t_{u}}-a e^{T_{I F}}+a}\right] .
\end{aligned}
$$

For any given $\tau$, the solution of variables $T_{u}, t_{u p}, \Delta t_{u}$ and $\tau_{u}$ uniquely determines the periodic pattern. On the other hand, we can calculate the minimum value $\tau_{\min }$ subject to the condition where $t_{u p}=0$ and the maximum value $\tau_{\max }$ subject to the condition where $t_{u p}=T_{A \vartheta}$. The segment between $A$ and $B$ in Figure 5 represents the interval for the existence of the periodic pattern $\left(1 W_{u}\right)$ with $\sigma=\left(2 W_{u}\right)$.

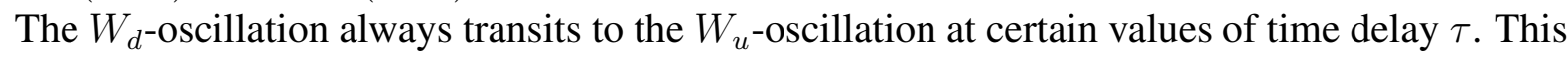
transition implies that any periodic pattern in the case where $R=N-1$ contains at most one $W_{d}$-oscillation. We now discuss the periodic patterns $\left(1 W_{d}, h W_{u}\right)$ with $h \geq 1$. Condition (4.6) immediately gives rise to

Theorem 7. (i) The periodic patterns $\left(1 W_{d}, h W_{u}\right)$ with $h \geq 1$ can be generated if and only if

$$
\left\{\begin{array}{l}
\tau=h T+T_{F R}+(h-1) T_{I F}+h\left(t_{u p}+\Delta t_{u}\right)+t_{\text {down }}-\tau_{u} \\
\Delta t_{u}-\tau_{u}=\Delta t_{d}-\tau_{d}
\end{array}\right.
$$

The minimum value of $\tau$ for the existence of such a periodic pattern is given by

$$
\tau_{\min }=N T+T_{F R}+(h-1) T_{I F}+\min _{\Delta t_{d}^{\min } \leq \Delta t_{d} \leq \Delta t_{\max }}\left[h\left(t_{u p}+\Delta t_{u}\right)+t_{\text {down }}-\tau_{u}\right],
$$

subject to the condition $\Delta t_{u}-\tau_{u}=\Delta t_{d}-\tau_{d}$. The maximum value of $\tau$ for the existence of such a periodic pattern is given by

$$
\tau_{\max }=h T+T_{F R}+h\left(T_{I F}+\Delta t_{\max }\right)-\left.\tau_{u}\right|_{\Delta t_{\max }},
$$

where $\left.\tau_{u}\right|_{\Delta t_{\max }}$ is defined in Theorem 6.

(ii) If $\tau$ reaches its minimum value at $\Delta t=\Delta t_{\text {max }}$, such periodic patterns can not be generated.

(iii) If $\tau$ reaches its minimum at $\Delta t<\Delta t_{\text {max }}$, then a pattern transition occurs at $\tau_{\text {max }}$.

Now we apply the above theorem to the periodic pattern $\sigma=\left(W_{u}, W_{d}\right)$, as shown in Figure 1 (a). The periods of the $W_{u}$-oscillation and the $W_{d}$-oscillation, condition (4.6), the additional time 


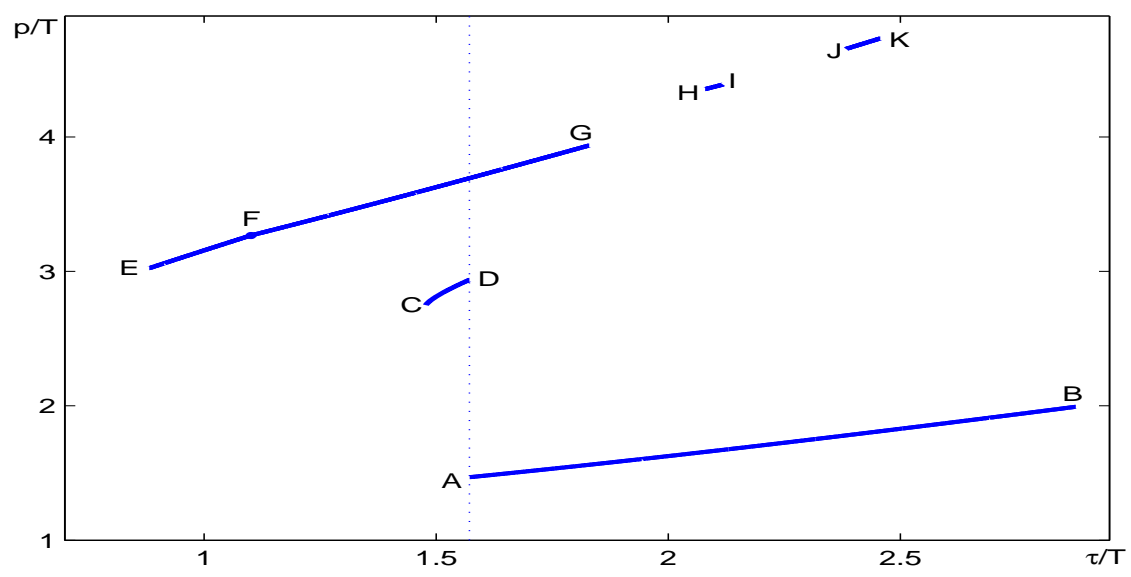

Figure 5: Plot of $p / T$ versus $\tau / T$ of six periodic patterns where $p$ is the period of a pattern: $\left(W_{u}\right)$ with $\sigma=\left(2 W_{u}\right)$ from $A$ to $B$; $\left(W_{u} W_{d}\right)$ from $C$ to $D ;\left(W_{d u} V\right)$ from $E$ to $F$; $\left(W_{u u} V\right)$ from $F$ to $G$; $\left(W_{d u} W_{d} V\right)$ from $H$ to $I$; and $\left(W_{u u} W_{d} V\right)$ from $J$ to $K$. The periodic pattern $\left(W_{d u} V\right)$ transits to the periodic pattern $\left(W_{u u} V\right)$ at $\mathrm{F}$ and the periodic pattern $\left(W_{u} W_{d}\right)$ transits to the periodic pattern $\left(W_{u}\right)$ with $\sigma=\left(2 W_{u}\right)$ at the dotted line.

delay (4.4), equations (5.1) and (5.2) yield

$$
\begin{aligned}
& T_{u}=T+t_{u p}+T_{I F}+\Delta t_{u}, \quad T_{d}=T+t_{\text {down }}+\Delta t_{d}, \\
& T_{d}+T_{F R}+t_{u p}=\tau+\tau_{d}, \quad \Delta t_{u}-\tau_{u}=\Delta t_{d}-\tau_{d}, \\
& \tau_{u}=\log \left[\frac{b\left(b-\vartheta-V_{A} e^{-T_{d}+T_{F R}}\right)}{\left.(b-\vartheta)^{2}-V_{A}^{2} e^{-T_{d}-T_{u}+2 T_{F R}}\right],}\right. \\
& \tau_{d}=\log \left[\frac{b\left(b-\vartheta-V_{A} e^{-T_{u}+T_{F R}}\right)}{(b-\vartheta)^{2}-V_{A}^{2} e^{-T_{d}-T_{u}+2 T_{F R}}}\right], \\
& t_{u p}=\log \left[\frac{I_{0}-V_{A}}{\left(I_{0}-V_{A}\right) e^{T_{I F}+\Delta t_{u}}-a e^{T_{I F}}+a}\right], \quad t_{\text {down }}=\log \left[\frac{I_{0}-V_{A}-a}{\left(I_{0}-V_{A}\right) e^{\Delta t_{d}}-a}\right] .
\end{aligned}
$$

For any given $\tau$, the solution of the above system uniquely determines the periodic pattern. On the other hand, we can calculate the minimum value $\tau_{\min }$ subject to $t_{\text {down }}=T_{I F}-T_{F R}$ and the maximum value $\tau_{\max }$ subject to $t_{\text {down }}=T_{I F}$. The segment between $C$ and $D$ in Figure 5 represents the interval for the existence of the periodic pattern $\left(W_{u}, W_{d}\right)$. The periodic pattern $\left(W_{u}, W_{d}\right)$ transits to the periodic pattern $\left(W_{u}\right)$ with $\sigma=\left(2 W_{u}\right)$ at the dotted line in Figure 5.

\subsection{Self-Inhibitory Periodic Patterns}

Self-inhibitory periodic patterns are composed of a $W_{c}^{m}$-oscillation and $(m-1) V$-oscillations, so these periodic patterns can be expressed as $\left(W_{d u . . u}^{m},(m-1) V\right)$ or $\left(W_{u . . u}^{m},(m-1) V\right)$. The spike emitted by the $W_{c}$-oscillation along with spikes emitted by the $V$-oscillations generate the 
$W_{c}$-oscillation. Examples of self-inhibitory periodic patterns are $\left(W_{u u} V\right)$ in Figure 1 (b) and $\left(W_{\text {иии }} 2 V\right)$ in Figure $2(\mathrm{c})$.

Lemma 8. For the self-inhibitory pattern $\left(W_{c}^{m},(m-1) V\right)$, the following holds:

(i) $t_{u p}^{c, 1}=\ldots=t_{u p}^{c, m-2}=T-T_{I F}, t_{u p}^{c, m-1}=T-T_{I F}+\tau_{v}-\tau_{c}$ for a $W_{d u \ldots u}^{m}$ oscillation, and the period of $W_{d u \ldots u}^{m}$ oscillation is $T_{d u \ldots u}^{(m)}=m T+t_{d o w n}^{c}+\Delta t_{c}+\tau_{v}-\tau_{c}$;

(ii) $t_{u p}^{c, 2}=\ldots=t_{u p}^{c, m-1}=T-T_{I F}, t_{u p}^{c, m}=T-T_{I F}+\tau_{v}-\tau_{c}$ for a $W_{u \ldots u}^{m}$-oscillation, and the period of $W_{u \ldots u}^{m}$-oscillation is $T_{u \ldots u}^{(m)}=m T+t_{u p}^{c, 1}+\Delta t_{c}+\tau_{v}-\tau_{c}$.

Proof: We consider the pattern $\sigma=\left(\pi_{1}, \pi_{2}, \ldots, \pi_{m}\right)=\left(W_{c},(m-1) V\right)$. For the $W_{c}$-oscillation, the evolution of the potential is characterized by $\left(t_{u p}^{c, i}, T_{I F}\right)$ between two consecutive self-feedbacks due to $\pi_{i}$ and $\pi_{i+1}$. Without the consideration of the additional delay, we have $t_{u p}^{c, i}+T_{I F}=T$ due to the oscillation $\pi_{i}=V$. The effect of the additional delay leads to $t_{u p}^{c, i}+T_{I F}+\tau_{i+1}-\tau_{i}=T$. In the situation where $\pi_{i}=\pi_{i+1}=V$, we still have $t_{u p}^{c, i}+T_{I F}=T$ since $\tau_{i}=\tau_{i+1}$. On the other hand, in the situation where $\pi_{i}=V$ and $\pi_{i+1}=W_{c}$ (it happens when $i=m-1$ for the $W_{d u \ldots u}^{m}$-oscillation and $i=m$ for the $W_{u \ldots u}^{m}$-oscillation), we have $t_{u p}^{c, i}+T_{I F}+\tau_{c}-\tau_{v}=T$. This completes the proof.

We now consider the periodic pattern $\left(W_{d u} V\right)$. The $W_{d u}$-oscillation is characterized by $\left(t_{d o w n}^{c}, T-\right.$ $\left.T_{I F}+\tau_{d u}-\tau_{v}, T_{I F}, \Delta t_{c}\right)$ and the corresponding additional delay is denoted by $\tau_{d u}$. Following the evolution of the potential of the $W_{d u}$-oscillation (similarly to the calculation in Lemma 3 ), we establish the relationship of $t_{\text {down }}^{c}$ and $\Delta t_{c}$.

$$
\Delta t_{c}=\log \left[e^{-T-t_{d o w n}^{c}-\tau_{d u}+\tau_{v}}+a \frac{1-e^{-T_{I F}}+e^{-T-\tau_{d u}+\tau_{v}}\left(1-e^{-t_{\text {down }}^{c}}\right)}{I_{0}-V_{A}}\right]
$$

When the self-feedback of the $W_{d u}$-oscillation is delivered, the potential is given by

$$
V_{2}=I_{0}-\left[\left(I_{0}-V_{A}\right) e^{-t_{\text {down }}^{c}}+a\left(1-e^{-t_{\text {down }}^{c}}\right)\right] e^{-T+T_{I F}-\tau_{d u}+\tau_{v}} .
$$

The period of $W_{d u}$-oscillation, condition (4.6) and additional delays (4.4) yield the following system

$$
\begin{aligned}
& T_{d u}=2 T+t_{\text {down }}^{c}+\Delta t_{c}+\tau_{v}-\tau_{d u}, \\
& T_{F R}+t_{\text {down }}^{c}+T-T_{I F}=\tau+\tau_{d u} \\
& \tau_{d u}=\log \left[\frac{b\left(b-\vartheta-V_{A} e^{-T+T_{F R}}\right)}{(b-\vartheta)^{2}-V_{A}^{2} e^{-T-T_{d u}+2 T_{F R}}}\right], \\
& \tau_{v}=\log \left[\frac{b\left(b-\vartheta-V_{A} e^{-T_{d u}+T_{F R}}\right)}{(b-\vartheta)^{2}-V_{A}^{2} e^{-T-T_{d u}+2 T_{F R}}}\right], \\
& \Delta t_{c}=\log \left[e^{-T-t_{d o w n}^{c}-\tau_{d u}+\tau_{v}}+a \frac{1-e^{-T_{I F}}+e^{-T-\tau_{d u}+\tau_{v}}\left(1-e^{\left.-t_{\text {down }}^{c}\right)}\right.}{I_{0}-V_{A}}\right] .
\end{aligned}
$$


Lemma 9. For the self-inhibitory periodic pattern $\left(W_{d u}, V\right)$, the minimum value of $\tau$ for the existence of such a periodic pattern is $\tau_{\min }=T+T_{F R}-T_{I F}+\left(t_{d o w n}^{c}-\tau_{d u}\right)_{\min }$, where $\left(t_{\text {down }}^{c}-\tau_{d u}\right)_{\min }$ is the minimum value of $t_{\text {down }}^{c}-\tau_{d u}$ of system $(5.10-5.14)$ subject to $t_{\text {down }}^{c}=T_{I F}-T_{F R}$ and the condition where $V_{2}=\vartheta$ of equation (5.9); the maximum value is $\tau_{\max }=T+T_{F R}-\left.\left(\tau_{d u}\right)\right|_{t_{d o w n}^{c}}=T_{I F}$ where $\left.\left(\tau_{d u}\right)\right|_{t_{\text {down }}^{c}=T_{I F}}$ is the solution of $\tau_{d u}$ of system $(5.10-5.14)$ subject to $t_{\text {down }}^{c}=T_{I F}$. On the other hand, for any given value $\tau \in\left(\tau_{\min }, \tau_{\max }\right)$, the solution of system $(5.10-5.14)$ uniquely determines the periodic pattern. Furthermore, the periodic pattern $\left(W_{d u}, V\right)$ transits to the periodic pattern $\left(W_{u u}, V\right)$ at $\tau_{\text {max }}$.

We then consider the periodic pattern $\left(W_{u u} V\right)$. The $W_{u u}$-oscillation is characterized by $\left(t_{u p}^{c, 1}, T_{I F}, T-\right.$ $\left.T_{I F}+\tau_{u u}-\tau_{v}, T_{I F}, \Delta t_{c}\right)$ and the corresponding additional delay is denoted by $\tau_{u u}$. Following the evolution of the potential of the $W_{u u}$-oscillation, we establish the relationship of $t_{u p}^{c, 1}$ and $\Delta t_{c}$. obtain

$$
\Delta t_{c}=\log \left[e^{-T-T_{I F}-t_{u p}^{c, 1}-\tau_{u u}+\tau_{v}}+\frac{a\left(1-e^{-T_{I F}}\right)\left(1+e^{-T-\tau_{u u}+\tau_{v}}\right)}{I_{0}-V_{A}}\right] .
$$

When the self-feedback of the $W_{d u}$-oscillation is delivered, the potential is given by

$$
V_{2}=I_{0}-\left[\left(I_{0}-V_{A}\right) e^{-t_{u p}^{c, 1}}+a\left(e^{T_{I F}}-1\right)\right] e^{-T-\tau_{u u}+\tau_{v}} .
$$

The period of $W_{u u}$-oscillation, condition (4.6), additional time delays (4.4) yield the following system

$$
\begin{aligned}
& T_{u u}=2 T+t_{u p}^{c, 1}+T_{I F}+\Delta t_{c}+\tau_{v}-\tau_{u u}, \\
& T_{F R}+t_{u p}^{c, 1}+T=\tau+\tau_{u u}, \\
& \tau_{u u}=\log \left[\frac{b\left(b-\vartheta-V_{A} e^{-T+T_{F R}}\right)}{\left.(b-\vartheta)^{2}-V_{A}^{2} e^{-T-T_{u u}+2 T_{F R}}\right],}\right. \\
& \tau_{v}=\log \left[\frac{b\left(b-\vartheta-V_{A} e^{-T_{u u}+T_{F R}}\right)}{(b-\vartheta)^{2}-V_{A}^{2} e^{-T-T_{u u}+2 T_{F R}}}\right], \\
& \Delta t_{c}=\log \left[e^{-T-T_{I F}-t_{u p}^{c, 1}-\tau_{u u}+\tau_{v}}+\frac{a\left(1-e^{-T_{I F}}\right)\left(1+e^{-T-\tau_{u u}+\tau_{v}}\right)}{I_{0}-V_{A}}\right] .
\end{aligned}
$$

Lemma 10. For the self-inhibitory periodic pattern $\left(W_{u u}, V\right)$, the minimum value of $\tau$ for the existence of such a periodic pattern is $\tau_{\min }=T+T_{F R}-\left.\left(\tau_{u u}\right)\right|_{t_{u p}^{c, 1}=0}$, where $\left.\left(\tau_{u u}\right)\right|_{t_{u p}^{c, 1}=0}$ is the solution of $\tau_{u u}$ of system (5.16) - (5.20) subject to $t_{u p}^{c, 1}=0$; the maximum value of $\tau$ is $\tau_{\max }=$ $T+T_{F R}+\left.\left(t_{u p}^{c, 1}-\tau_{u u}\right)\right|_{V_{2}}$, where $\left.\left(t_{u p}^{c, 1}-\tau_{u u}\right)\right|_{V_{2}}$ is the value of $t_{u p}^{c, 1}-\tau_{u u}$ of system $(5.16)-(5.20)$ subject to $V_{2}=\vartheta$ in equation (5.15). On the other hand, for any given value $\tau \in\left(\tau_{\min }, \tau_{\max }\right)$, the solution of system (5.16 - 5.20) uniquely determines the periodic pattern.

The segment between $E$ and $F$ in Figure 5 represents the interval for the existence of the periodic pattern $\left(W_{d u}, V\right)$. The segment between $F$ and $G$ in Figure 5 represents the interval for the existence of the periodic pattern $\left(W_{u u}, V\right)$. The periodic pattern $\left(W_{d u}, V\right)$ transits to the periodic pattern $\left(W_{u u}, V\right)$ at $F$. Some other self-inhibitory periodic patterns such as $\left(W_{d u u}, 2 V\right)$ and $\left(W_{u u u}, 2 V\right)$ will be presented in our case study. 


\subsection{Nearest-Neighbor-Inhibitory Periodic Patterns}

The simple nearest-neighbor-inhibitory periodic patterns are $\left(W_{c}^{m}, W_{d},(m-1) V\right)$ with $m \geq 2$ such as $\left(W_{d u} W_{d} V\right)$ and $\left(W_{u u} W_{d} V\right)$. For the periodic pattern $\left(W_{c}^{m}, W_{d},(m-1) V\right)$, the inhibitory self-feedback due to the $W_{c}^{m}$-oscillation generates the $W_{d}$-oscillation that in turn, along with the $V$-oscillations, generates the $W_{c}^{m}$-oscillation. Examples of nearest-neighbor-inhibitory periodic patterns are $\left(W_{u u} W_{d} V\right)$ and $\left(W_{u u} W_{u} V W_{u}\right)$ in Figure 2.

We first consider the periodic pattern $\left(W_{d u} W_{d} V\right)$. The $W_{d}$-oscillation is characterized by $\left(t_{\text {down }}, \Delta t_{d}\right)$ and the $W_{d u}$-oscillation is characterized by $\left(t_{d o w n}^{c}, t_{u p}^{c, 1}, T_{I F}, \Delta t_{c}\right)$. For the $W_{d u}$-oscillation, when the self-feedback due to the $V$-oscillation arrives, the potential is given by

$$
V_{2}=I_{0}-\left(I_{0}-V_{A}\right) e^{-t_{u p}^{c, 1}-t_{d o w n}^{c}}-a\left(1-e^{-t_{d o w n}^{c}}\right) e^{-t_{u p}^{c, 1}} .
$$

The $W_{d u}$-oscillation yields

$$
\begin{aligned}
T_{d u} & =T+t_{\text {down }}^{c}+t_{\text {up }}^{c, 1}+T_{I F}+\Delta t_{c} \\
\Delta t_{c} & =\log \left[e^{-T_{I F}-t_{\text {down }}^{c}-t_{u p}^{c, 1}}+a \frac{1-e^{-T_{I F}}+\left(1-e^{-t_{\text {down }}^{c}}\right) e^{-t_{u p}^{c, 1}-T_{I F}}}{I_{0}-V_{A}}\right] .
\end{aligned}
$$

The $W_{d^{-}}$oscillation leads to

$$
\begin{aligned}
& T_{d}=T+t_{\text {down }}+\Delta t_{d} \\
& t_{\text {down }}=\log \left[\frac{I_{0}-V_{A}-a}{\left(I_{0}-V_{A}\right) e^{\Delta t_{d}}-a}\right] .
\end{aligned}
$$

Condition (4.6) gives rise to

$$
\begin{aligned}
& 2 T+t_{\text {down }}+\Delta t_{d}+T_{F R}+t_{\text {down }}^{c}=\tau+\tau_{d}+T_{I F}, \\
& t_{\text {up }}^{c, 1}=T+t_{\text {down }}+\Delta t_{d}-T_{I F}-\tau_{d}+\tau_{v}, \\
& \Delta t_{c}+t_{\text {down }}-\tau_{\text {du }}+\tau_{v}=0 .
\end{aligned}
$$

Additional delays are given by

$$
\begin{aligned}
& \tau_{d u}=\log \left\{\frac{b\left[(b-\vartheta)\left(b-\vartheta-V_{A} e^{-T+T_{F R}}\right)+V_{A}^{2} e^{-T_{d}-T+2 T_{F R}}\right]}{\left.(b-\vartheta)^{3}+V_{A}^{3} e^{-T_{d u}-T_{d}-T+3 T_{F R}}\right\},}\right. \\
& \tau_{d}=\log \left\{\frac{b\left[(b-\vartheta)\left(b-\vartheta-V_{A} e^{-T_{d u}+T_{F R}}\right)+V_{A}^{2} e^{-T_{d u}-T+2 T_{F R}}\right]}{\left.(b-\vartheta)^{3}+V_{A}^{3} e^{-T_{d u}-T_{d}-T+3 T_{F R}}\right\},}\right. \\
& \tau_{v}=\log \left\{\frac{b\left[(b-\vartheta)\left(b-\vartheta-V_{A} e^{-T_{d}+T_{F R}}\right)+V_{A}^{2} e^{-T_{d u}-T_{d}+2 T_{F R}}\right]}{\left.(b-\vartheta)^{3}+V_{A}^{3} e^{-T_{d u}-T_{d}-T+3 T_{F R}}\right\} .}\right.
\end{aligned}
$$

Lemma 11. For the nearest-neighbor-inhibitory periodic pattern $\left(W_{d u} W_{d} V\right)$, the minimum value of $\tau$ for the existence of such a periodic pattern is $\tau_{\text {min }}=2 T+T_{F R}-T_{I F}+\left(t_{\text {down }}+t_{\text {down }}^{c}+\Delta t_{d}-\right.$ $\left.\tau_{d}\right)_{\max }$, where $\left(t_{\text {down }}+t_{\text {down }}^{c}+\Delta t_{d}-\tau_{d}\right)_{\text {max }}$ is the maximum value of $t_{\text {down }}+t_{\text {down }}^{c}+\Delta t_{d}-\tau_{u}$ 
of system $(5.22)-(5.31)$ subject to $t_{\text {down }}^{c}=T_{I F}-T_{F R}$ or $t_{\text {down }}^{c}=T_{I F}$ or $V_{2}=\vartheta$ of equation (5.21); the maximum value of $\tau$ is $\tau_{\max }=T+T_{F R}-\left.\left(t_{\text {down }}+t_{\text {down }}^{c}+\Delta t_{d}-\tau_{d}\right)\right|_{\text {min }}$, where $\left.\left(t_{\text {down }}+t_{\text {down }}^{c}+\Delta t_{d}-\tau_{d}\right)\right|_{\text {min }}$ is the minimum value of $t_{\text {down }}+t_{\text {down }}^{c}+\Delta t_{d}-\tau_{d}$ of system $(5.22)-(5.31)$ subject to $t_{d o w n}^{c}=T_{I F}$ or $t_{d o w n}=T_{I F}-T_{F R}$. On the other hand, for any given value $\tau \in\left(\tau_{\min }, \tau_{\max }\right)$, the solution of system (5.22) - (5.31) uniquely determines the periodic pattern.

We then consider the periodic pattern $\left(W_{u u} W_{d} V\right)$. The $W_{d}$-oscillation is characterized by

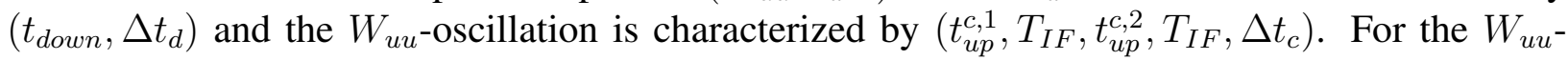
oscillation, when the self-feedback due to the $V$-oscillation arrives, the potential is given by

$$
V_{2}=I_{0}-\left(I_{0}-V_{A}\right) e^{-t_{u p}^{c, 1}-t_{u p}^{c, 2}-T_{I F}}-a\left(1-e^{-T_{I F}}\right) e^{-t_{u p}^{c, 2}}
$$

The $W_{u u}$-oscillation yields

$$
\begin{aligned}
& T_{u u}=T+t_{u p}^{c, 1}+t_{u p}^{c, 2}+2 T_{I F}+\Delta t_{c}, \\
& \Delta t_{c}=\log \left[e^{-2 T_{I F}-t_{u p}^{c, 1}-t_{u p}^{c, 2}}+a \frac{\left(1-e^{-T_{I F}}\right)\left(1+e^{-t_{u p}^{c, 2}-T_{I F}}\right)}{I_{0}-V_{A}}\right] .
\end{aligned}
$$

The $W_{d}$-oscillation leads to

$$
\begin{aligned}
& T_{d}=T+t_{\text {down }}+\Delta t_{d} \\
& t_{\text {down }}=\log \left[\frac{I_{0}-V_{A}-a}{\left(I_{0}-V_{A}\right) e^{\Delta t_{d}}-a}\right] .
\end{aligned}
$$

Condition (4.6) gives rise to

$$
\begin{aligned}
& 2 T+t_{\text {down }}+\Delta t_{d}+T_{F R}+t_{\text {up }}^{c, 1}=\tau+\tau_{d}, \\
& t_{\text {up }}^{c, 2}=T+t_{\text {down }}+\Delta t_{d}-T_{I F}-\tau_{d}+\tau_{v}, \\
& \Delta t_{c}+t_{\text {down }}-\tau_{\text {uu }}+\tau_{v}=0 .
\end{aligned}
$$

Additional delays are given by

$$
\begin{aligned}
& \tau_{u u}=\log \left\{\frac{b\left[(b-\vartheta)\left(b-\vartheta-V_{A} e^{-T+T_{F R}}\right)+V_{A}^{2} e^{-T_{d}-T+2 T_{F R}}\right]}{\left.(b-\vartheta)^{3}+V_{A}^{3} e^{-T_{u u}-T_{d}-T+3 T_{F R}}\right\},}\right. \\
& \tau_{d}=\log \left\{\frac{b\left[(b-\vartheta)\left(b-\vartheta-V_{A} e^{-T_{u u}+T_{F R}}\right)+V_{A}^{2} e^{-T_{u u}-T+2 T_{F R}}\right]}{\left.(b-\vartheta)^{3}+V_{A}^{3} e^{-T_{u u}-T_{d}-T+3 T_{F R}}\right\},}\right. \\
& \tau_{v}=\log \left\{\frac{b\left[(b-\vartheta)\left(b-\vartheta-V_{A} e^{-T_{d}+T_{F R}}\right)+V_{A}^{2} e^{-T_{u u}-T_{d}+2 T_{F R}}\right]}{\left.(b-\vartheta)^{3}+V_{A}^{3} e^{-T_{u u}-T_{d}-T+3 T_{F R}}\right\} .}\right.
\end{aligned}
$$

Lemma 12. For the the nearest-neighbor-inhibitory periodic pattern $\left(W_{u u} W_{d} V\right)$, the minimum value of $\tau$ for the existence of such a periodic pattern is $\tau_{\text {min }}=2 T+T_{F R}+\left.\left(t_{\text {down }}+\Delta t_{d}-\tau_{d}\right)\right|_{\text {max }}$, where $\left.\left(t_{\text {down }}+\Delta t_{d}-\tau_{d}\right)\right|_{\text {max }}$ is the maximum value of $t_{\text {down }}+\Delta t_{d}-\tau_{d}$ of system (5.33) $-(5.42)$ 
subject to $t_{u p}^{c, 1}=0$ or $t_{\text {down }}^{c}=T_{I F}-T_{F R}$; the maximum value of $\tau$ is $\tau_{\text {max }}=2 T+T_{F R}+\left(t_{\text {down }}+\right.$ $\left.t_{u p}^{c, 1}+\Delta t_{d}-\tau_{d}\right)_{\text {min }}$, where $\left(t_{\text {down }}+t_{u p}^{c, 1}+\Delta t_{d}-\tau_{d}\right)_{\text {min }}$ is the minimum value of $t_{\text {down }}+t_{\text {up }}^{c, 1}+\Delta t_{d}-\tau_{d}$ of system (5.33) - (5.42) subject to $t_{\text {down }}^{c}=T_{I F}$ or $V_{2}=\vartheta$ of equation (5.32). On the other hand, for any given value $\tau \in\left(\tau_{\min }, \tau_{\max }\right)$, the solution of system (5.33) - (5.42) uniquely determines the periodic pattern.

The segment between $H$ and $I$ in Figure 5 represents the interval for the existence of the periodic pattern $\left(W_{d u} W_{d} V\right)$ and the segment between $J$ and $K$ represents the interval for the existence of the periodic pattern $\left(W_{u u} W_{d} V\right)$.

Other possible nearest-neighbor-inhibitory periodic patterns are $\left(W_{c}^{m}, W_{u},(m-1) V, W_{d}\right)$ and $\left(W_{c}^{m}, W_{u},(m-1) V, W_{u}\right)$ with $m \geq 2$. For example, for the periodic pattern $\left(W_{c}^{m}, W_{u},(m-\right.$ 1) $\left.V, W_{d}\right)$, the inhibitory self-feedback delivered by the $W_{c}^{m}$-oscillation generates the $W_{u}$-oscillation, then the feedback from the $W_{u}$-oscillation generates the $W_{d}$-oscillation, and the feedbacks from the $V$-oscillations and the $W_{d}$-oscillation generate the $W_{c}^{m}$-oscillation. At a certain critical value of the delay, the periodic pattern $\left(W_{c}^{m}, W_{u},(m-1) V, W_{d}\right)$ will transit to the periodic pattern $\left(W_{c}^{m}, W_{u},(m-1) V, W_{u}\right)$. The calculation similar to the periodic pattern $\left(W_{u u} W_{d} V\right)$ can apply to periodic patterns $\left(W_{c}^{m}, W_{u},(m-1) V, W_{d}\right)$ and $\left(W_{c}^{m}, W_{u},(m-1) V, W_{u}\right)$, but it is quite complicated. Hence, we only present some numerical results in our case study (Section 6).

\subsection{Far-Range-Inhibitory Periodic Patterns}

If the inhibitory feedback delivered by the $W_{c}$-oscillation affects an action potential which is far away from its nearest neighbor, the configuration of the patterns becomes more complicated. For example, multiple $W_{c}$-oscillations may coexist in one periodic pattern. However, because of the complicated interaction within such a periodic pattern, it may take very long time to converge to such a pattern from an initial condition. This long recognition time prevents these patterns from potential applications in neural information transmission or object recognition. Hence, we shall not conduct a detailed discussion, though we shall list some patterns in our case study in the next section.

\section{A Case Study}

We now apply the general results obtained in the previous section to a special case to illustrate the detailed periodic patterns and the occurrence of coexisting multiple patterns. The parameter values are identical to those used in Subsection 2.1, i.e., $\vartheta=1 \mathrm{mV}, I_{0}=1.45 \mu \mathrm{A}, a=2.7 \mu \mathrm{A}$, $b=6 \mu A, T_{I F}=0.5 \mathrm{~ms}, T_{F R}=0.35 \mathrm{~ms}, c=3.5 \mathrm{mV}$ and $V_{A}=-0.1187 \mathrm{mV}, T_{A \vartheta}=1.2488 \mathrm{~ms}$, $T=1.5988 \mathrm{~ms}$.

Corresponding to each spike of the excitatory neuron, there is always an inhibitory self-feedback (SFB) to act on the excitatory neuron in a time delay $\tau^{\prime}=\tau+\tau_{A}$, where $\tau_{A}$ only depends on the basic oscillations of the excitatory neuron. However, in what follows, we take $\tau^{\prime}=\tau+0.2$ to discuss the periodic patterns exhibited by the excitatory neuron in the interval $\tau^{\prime} \in[n T, n T+T)$ with 
a positive integer $n$. Value 0.2 is only an empirical constant but periodic patterns do not depend on this value.

We now introduce an important quantity, the average time of convergence to a pattern, as a measurement of recognition of this pattern. The numerical calculation of this average convergence time is implemented in the following way. We consider a periodic pattern $\sigma=\left(\pi_{1}, \ldots, \pi_{k}\right)$ with $\pi_{k} \in\left(V, W_{d}, W_{u}, W_{c}\right)$, which has the period $p$ and consists of $k$ action potentials. For any solution of neuron $\mathrm{E}$ in the model (2.1), we denote the firing times by $r_{1}, r_{2}, \ldots$ for $t>0$, the corresponding action potentials by $\left\{\tilde{\pi}_{1}, \tilde{\pi}_{2}, \ldots\right\}$. For each firing time $r_{n}(\mathrm{n}=1,2, \ldots)$, we calculate the duration of $k$ consecutive action potentials $\left\{\tilde{\pi}_{n}, \ldots, \tilde{\pi}_{n+k-1}\right\}$ as $p_{n}=\sum_{i=n}^{n+k-1}\left(r_{i+1}-r_{i}\right)$, and calculate the relative error $\frac{\left|p_{n}-p\right|}{p}$. Then we define the convergence time of this periodic pattern by $r_{n}$ for the first $n$ whose relative error is less than a given tolerate $\left(10^{-5}\right.$ in our study) and express it with respect to the period $p$ of the pattern, i.e, $b=r_{n} / p$. The domain of attraction corresponding to the given periodic pattern should yield the convergent time in a range, denoted by $\left(b_{\min } p \sim b_{\max } p\right)$. Since the given periodic pattern actually exists in an interval $\tau \in\left(\tau_{\min }, \tau_{\max }\right)$, we denote $p_{\min }$ by the period $p$ corresponding to $\tau_{\min }$ and $p_{\max }$ by the period $p$ corresponding to $\tau_{\max }$. Then we define the average convergence time as $\frac{b_{\min }+b_{\max }}{2} \times \frac{p_{\min }+p_{\max }}{2}$ with respect to the intrinsic spiking period $T$. Note that these quantities, $b_{\min }, b_{\max }, p_{\min }$ and $p_{\max }$, are based on numerical calculation.
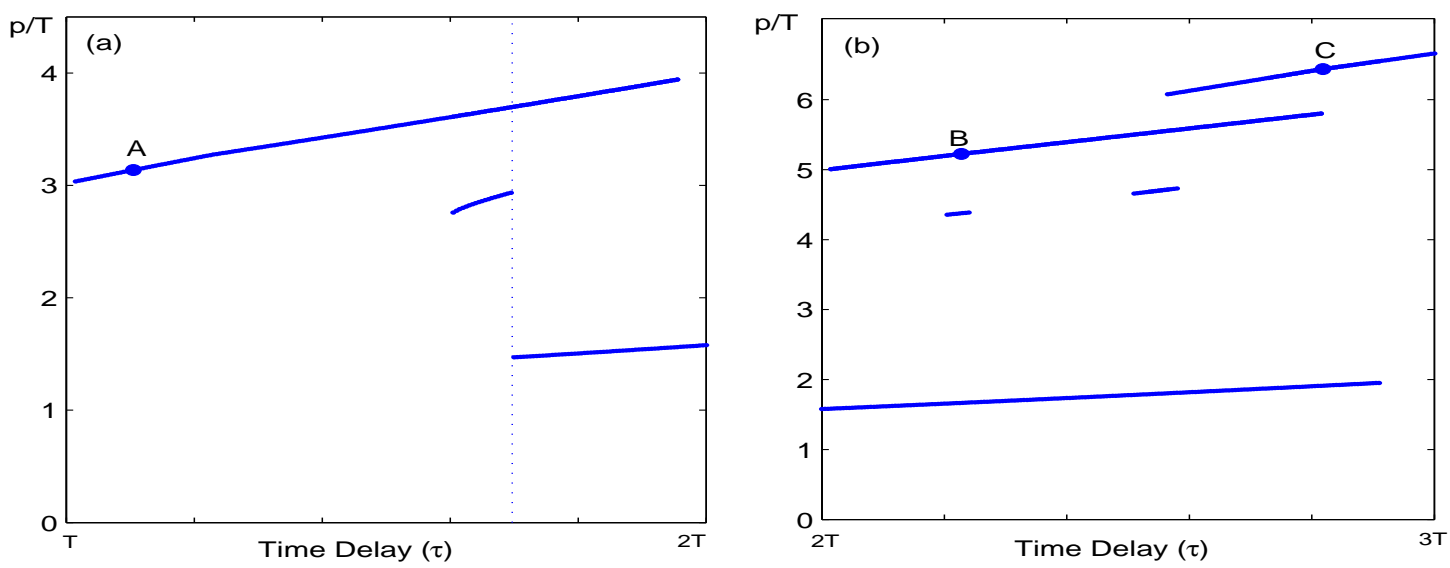

Figure 6: Plot of $p / T$ versus $\tau^{\prime}$ when $\tau^{\prime} \in\left[T, 2 T\right.$ ) (a) and $\tau^{\prime} \in[2 T, 3 T)$ (b), where $p$ is the minimum period of a given pattern in the specific interval and $T$ is the intrinsic spiking period of the excitatory neuron. (a) From the bottom to the top, periodic patterns are $\left(W_{u}\right)$ with $\sigma=$ $\left(2 W_{u}\right),\left(W_{u} W_{d}\right),\left(W_{d u} V\right)$ and $\left(W_{u u} V\right)$. The periodic pattern $\left(W_{d u} V\right)$ transits to the periodic pattern $\left(W_{u u} V\right)$ at $A$ and the periodic pattern $\left(W_{u} W_{d}\right)$ transits to the periodic pattern $\left(W_{u}\right)$ with $\sigma=$ $\left(2 W_{u}\right)$ at the dotted line. (b) From the bottom to the top, periodic patterns are $\left(W_{u}\right)$ with $\sigma=$ $\left(2 W_{u}\right),\left(W_{d u} W_{d} V\right),\left(W_{u u} W_{d} V\right),\left(W_{d u u} 2 V\right),\left(W_{u u u} 2 V\right),\left(W_{u u} W_{u} V W_{d}\right)$ and $\left(W_{u u} W_{u} V W_{u}\right)$. The periodic pattern $\left(W_{d u u} 2 V\right)$ transits to the periodic pattern $\left(W_{u u u} 2 V\right)$ at $B$ and the periodic pattern $\left(W_{u u} W_{u} V W_{d}\right)$ transits to the periodic pattern $\left(W_{u u} W_{u} V W_{u}\right)$ at $C$. 


\begin{tabular}{c|c|c|c|c}
\hline Pattern & Type & Interval of $\tau^{\prime}$ & $\begin{array}{c}\text { Convergence } \\
\text { Time }\end{array}$ & $\begin{array}{c}\text { Average Conver- } \\
\text { gence Time }\end{array}$ \\
\hline$\left(W_{u}\right)$ & $W_{u}$ & $(1.695 T, 2 T]$ & $20 p \sim 40 p$ & $51.33 \mathrm{~T}$ \\
\hline$\left(W_{d} W_{u}\right)$ & $W_{u}$ and $W_{d}$ & {$[1.605 T, 1.695 T]$} & $30 p \sim 80 p$ & $156.58 \mathrm{~T}$ \\
\hline$\left(W_{d u} V\right)$ & self-inhibitory & {$[1.015 T, 1.10 T)$} & $<1.8 p$ & $3.14 \mathrm{~T}$ \\
$\left(W_{u u} V\right)$ & self-inhibitory & $(1.10 T, 1.955 T]$ & $<1.8 p$ & $3.14 \mathrm{~T}$ \\
\hline
\end{tabular}

Table 1: Periodic patterns in the interval $\tau^{\prime} \in[T, 2 T)$, where $p$ is the period of a pattern for any given $\tau^{\prime}$ in the specific interval.

\subsection{Periodic Patterns on the Interval $\tau^{\prime} \in[T, 2 T)$}

On the interval $\tau^{\prime} \in[T, 2 T)$, there exist four periodic patterns, shown in Table 1 and Figure 6 (a): $\left(W_{u}\right)$ with $\sigma=\left(2 W_{u}\right),\left(W_{d} W_{u}\right)$, the self-inhibitory periodic patterns $\left(W_{d u} V\right)$ and $\left(W_{u u} V\right)$. Figure 1 shows the periodic patterns $\left(W_{d} W_{u}\right)$ and $\left(W_{u u} V\right)$. The periodic pattern $\left(W_{d u} V\right)$ transits to the periodic pattern $\left(W_{u u} V\right)$ at $A$ and the periodic pattern $\left(W_{u} W_{d}\right)$ transits to the periodic pattern $\left(W_{u}\right)$ with $\sigma=\left(2 W_{u}\right)$ at the dotted line in Figure 6 (a). Periodic patterns $\left(W_{u u} V\right)$ and $\left(W_{d} W_{u}\right)$ coexist on the interval $\tau^{\prime} \in[1.605 T, 1.695 T]$ and periodic patterns $\left(W_{u u} V\right)$ and $\left(W_{u}\right)$ coexist on the interval $\tau^{\prime} \in(1.695 T, 1.955 T]$. It follows easily from Table 1 that the self-inhibitory periodic patterns $\left(W_{d u} V\right)$ and $\left(W_{u u} V\right)$ have faster speed to be recognized than the periodic patterns $\left(W_{u}\right)$ and $\left(W_{u} W_{d}\right)$.

\subsection{Periodic Patterns on the Interval $\tau^{\prime} \in[2 T, 3 T)$}

On the interval $\tau^{\prime} \in[2 T, 3 T)$, there exist seven periodic patterns, shown in Table 2 and Figure 6 (b): $\left(W_{u}\right)$ with $\sigma=\left(2 W_{u}\right),\left(W_{d u} W_{d} V\right),\left(W_{u u} W_{d} V\right),\left(W_{d u u} 2 V\right),\left(W_{u u u} 2 V\right),\left(W_{u u} W_{u} V W_{d}\right)$ and $\left(W_{u u} W_{u} V W_{u}\right)$. In particular, self-inhibitory patterns $\left(W_{d u u} 2 V\right)$ and $\left(W_{u u u} 2 V\right)$ have faster recognition time than nearest-neighbor-inhibitory patterns $\left(W_{d u} W_{d} V\right),\left(W_{u u} W_{d} V\right)\left(W_{u u} W_{u} V W_{d}\right)$ and $\left(W_{u u} W_{u} V W_{u}\right)$. The periodic pattern $\left(W_{d u u} 2 V\right)$ transits to the periodic pattern $\left(W_{u u u} 2 V\right)$ at $B$ and the periodic pattern $\left(W_{u u} W_{u} V W_{d}\right)$ transits to the periodic pattern $\left(W_{u u} W_{u} V W_{u}\right)$ at $C$ in Figure 6 (b). The overlapping intervals present the coexistence of multiple periodic patterns, for example, Figure 2 shows the coexistence of periodic patterns $\left(W_{u}\right)$ with $\sigma=\left(2 W_{u}\right),\left(W_{u u} W_{d} V\right)$, $\left(W_{u u u} 2 V\right)$ and $\left(W_{u u} W_{u} V W_{u}\right)$ on the interval $\tau^{\prime} \in[2.205 T, 2.225 T]$.

\subsection{Periodic Patterns on the Interval $\tau^{\prime} \in[3 T, 4 T)$}

Figure 7 and Table 3 show periodic patterns on the interval $\tau^{\prime} \in[3 T, 4 T):\left(W_{u}\right)$ with $\sigma=$ $\left(3 W_{u}\right),\left(W_{\text {duи }} W_{d} 2 V\right),\left(W_{\text {иии }} W_{d} 2 V\right),\left(W_{\text {uи }} W_{u} V W_{u}\right),\left(W_{\text {duи }} 3 V\right),\left(W_{\text {ииии }} 3 V\right),\left(W_{\text {иии }} W_{u} 2 V W_{d}\right)$, $\left(W_{u u u} W_{u} 2 V W_{u}\right),\left(W_{u u} W_{d u} V W_{u} V W_{d u} W_{u u} V W_{d} V\right)$ and $\left(W_{u u} W_{u u} V W_{u} V W_{d u} W_{u u} V W_{d} V\right) . \mathrm{Pe}-$ riodic pattern $\left(W_{d u u} W_{d} 2 V\right)$ transits to the periodic pattern $\left(W_{u u u} W_{d} 2 V\right)$ at $E$ in Figure 7 ; the periodic pattern $\left(W_{\text {duuu }} 3 V\right)$ transits to the periodic pattern $\left(W_{\text {uиuи }} 3 V\right)$ at $F$; the periodic pattern $\left(W_{u u u} W_{u} 2 V W_{d}\right)$ transits to the periodic pattern $\left(W_{u u u} W_{u} 2 V W_{u}\right)$ at $G$; the periodic pattern 


\begin{tabular}{c|c|c|c|c}
\hline Pattern & Type & Interval of $\tau^{\prime}$ & $\begin{array}{c}\text { Convergence } \\
\text { Time }\end{array}$ & $\begin{array}{c}\text { Average Conver- } \\
\text { gence Time }\end{array}$ \\
\hline$\left(W_{u}\right)$ & $W_{u}$ & {$[2 T, 2.91 T]$} & $20 p \sim 40 p$ & $51.33 \mathrm{~T}$ \\
\hline$\left(W_{d u} W_{d} V\right)$ & nearest-neighbor & {$[2.205 T, 2.24 T]$} & $6 p \sim 10 p$ & $34.97 \mathrm{~T}$ \\
$\left(W_{u u} W_{d} V\right)$ & nearest-neighbor & {$[2.51 T, 2.58 T]$} & $3 p \sim 6 p$ & $21.12 \mathrm{~T}$ \\
\hline$\left(W_{d u u} 2 V\right)$ & self-inhibitory & {$[2.015 T, 2.225 T]$} & $<1 p$ & $2.56 \mathrm{~T}$ \\
$\left(W_{u u u} 2 V\right)$ & self-inhibitory & $(2.225 T, 2.815 T]$ & $<1 p$ & $2.76 \mathrm{~T}$ \\
\hline$\left(W_{u u} W_{u} V W_{d}\right)$ & nearest-neighbor & {$[2.565 T, 2.815 T]$} & $21 p-27 p$ & $104.5 \mathrm{~T}$ \\
$\left(W_{u u} W_{u} V W_{u}\right)$ & nearest-neighbor & $(2.815 T, 3 T)$ & $8 p-10 p$ & $104.5 \mathrm{~T}$ \\
\hline
\end{tabular}

Table 2: Periodic patterns in the interval $\tau^{\prime} \in[2 T, 3 T)$, where $p$ is the period of a periodic pattern.

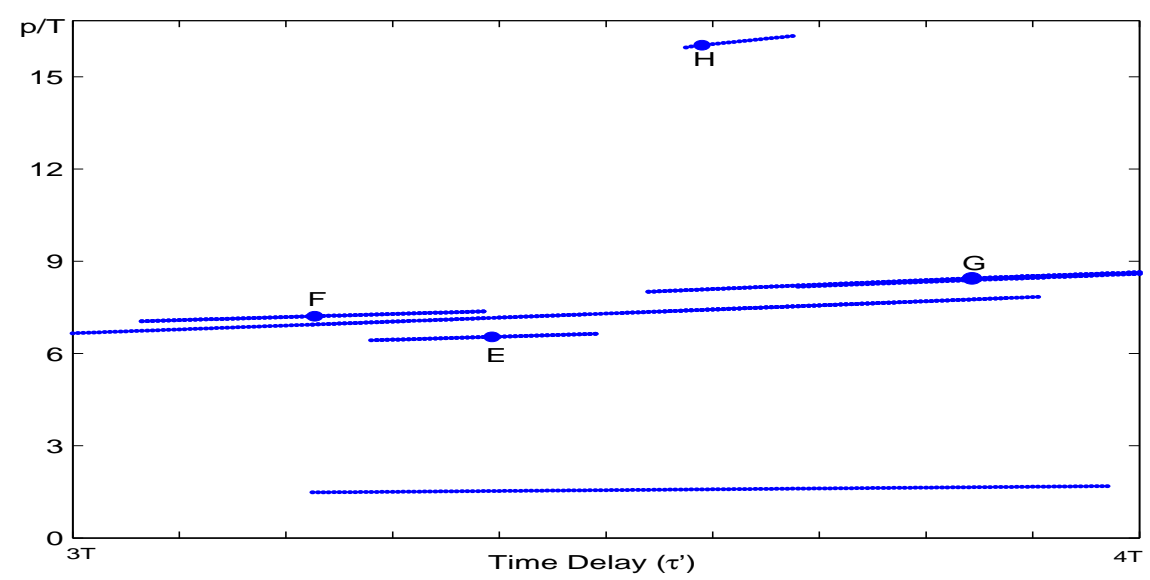

Figure 7: Plot of $p / T$ versus $\tau^{\prime}$ for $\tau^{\prime} \in[3 T, 4 T)$, where $p$ is the minimum period of a given pattern in the specific interval. From the bottom to the top, periodic patterns are $\left(W_{u}\right)$ with $\sigma=\left(3 W_{u}\right),\left(W_{\text {duu }} W_{d} 2 V\right),\left(W_{\text {uuu }} W_{d} 2 V\right), \quad\left(W_{u u} W_{u} V W_{u}\right)$, $\left(W_{\text {диии }} 3 V\right),\left(W_{\text {ииии }} 3 V\right),\left(W_{\text {uиu }} W_{u} 2 V W_{d}\right),\left(W_{\text {uиu }} W_{u} 2 V W_{u}\right),\left(W_{u и} W_{d u} V W_{u} V W_{d u} W_{u u} V W_{d} V\right)$ and $\left(W_{u u} W_{u u} V W_{u} V W_{d u} W_{u u} V W_{d} V\right)$. The periodic pattern $\left(W_{d u u} W_{d} 2 V\right)$ transits to the periodic pattern $\left(W_{\text {uuu }} W_{d} 2 V\right)$ at $E$; the periodic pattern $\left(W_{\text {duuu }} 3 V\right)$ transits to the periodic pattern $\left(W_{\text {uиuи }} 3 V\right)$ at $F$; the periodic pattern $\left(W_{\text {uиu }} W_{u} 2 V W_{d}\right)$ transits to the periodic pattern $\left(W_{\text {uuu }} W_{u} 2 V W_{u}\right)$ at $G$; the periodic pattern $\left(W_{u u} W_{d u} V W_{u} V W_{d u} W_{u u} V W_{d} V\right)$ transits to the periodic patterns $\left(W_{u u} W_{u u} V W_{u} V W_{d u} W_{u u} V W_{d} V\right)$ at $H$.

$\left(W_{u u} W_{d u} V W_{u} V W_{d u} W_{u u} V W_{d} V\right)$ transits to the periodic pattern $\left(W_{u u} W_{u u} V W_{u} V W_{d u} W_{u u} V W_{d} V\right)$ at $H$. The overlapping subintervals in Figure 7 presents the coexistence of multiple periodic patterns, for example, periodic patterns $\left(W_{u u} W_{u} V W_{u}\right)$ and $\left(W_{d u u u} 3 V\right)$ coexist on the interval $\tau^{\prime} \in$ $[3.065 T, 3.225 T]$. According to the average convergence time of periodic patterns listed in Table 3 , the self-inhibitory patterns have the fastest speed to be recognized; next is the nearest-neighborinhibitory patterns and the patterns composed of only basic $W_{u}$-oscillations and $W_{d}$-oscillation; 


\begin{tabular}{c|c|c|c|c}
\hline Pattern & Type & Interval of $\tau^{\prime}$ & $\begin{array}{c}\text { Convergence } \\
\text { Time }\end{array}$ & $\begin{array}{c}\text { Average Conver- } \\
\text { gence Time }\end{array}$ \\
\hline$\left(W_{u}\right)$ & $W_{u}$ & {$[3.225 T, 3.97 T]$} & $42 \sim 70 p$ & $89 \mathrm{~T}$ \\
\hline$\left(W_{d u u} W_{d} 2 V\right)$ & nearest-neighbor & {$[3.28 T, 3.39 T]$} & $2 \sim 5 p$ & $23 \mathrm{~T}$ \\
$\left(W_{u u u} W_{d} 2 V\right)$ & nearest-neighbor & $(3.39 T, 3.49 T]$ & $1 \sim 4 p$ & $16 \mathrm{~T}$ \\
\hline$\left(W_{\text {uu }} W_{u} V W_{u}\right)$ & nearest-neighbor & {$[3 T, 3.905 T]$} & $10 \sim 18 p$ & $102 \mathrm{~T}$ \\
\hline$\left(W_{\text {duuu }} 3 V\right)$ & self-inhibitory & {$[3.065 T, 3.225 T]$} & $<1 p$ & $4 \mathrm{~T}$ \\
$\left(W_{\text {uиuu }} 3 V\right)$ & self-inhibitory & $(3.225 T, 3.385 T]$ & $<1 p$ & $4 \mathrm{~T}$ \\
\hline$\left(W_{\text {uuu }} W_{u} 2 V W_{d}\right)$ & nearest-neighbor & {$[3.54 T, 3.84 T]$} & $24-40 p$ & $252 \mathrm{~T}$ \\
$\left(W_{\text {uuu }} W_{u} 2 V W_{u}\right)$ & nearest-neighbor & $(3.84 T, 4 T)$ & $8-10 p$ & $77 \mathrm{~T}$ \\
\hline$*$ & far-range & {$[3.575 T, 3.675 T]$} & $14-54 p$ & $549 \mathrm{~T}$ \\
\hline
\end{tabular}

Table 3: Periodic patterns in the interval $\tau^{\prime} \in[3 T, 4 T)$, where $p$ is the period of a pattern for any given $\tau^{\prime}$ in the specific interval, $*$ represents $\left(W_{u u} W_{d u} V W_{u} V W_{d u} W_{u u} V W_{d} V\right)$ and $\left(W_{u u} W_{u u} V W_{u} V W_{d u} W_{u u} V W_{d} V\right)$.

the slowest is the far-range-inhibitory patterns due to the complicated interaction among the basic oscillations. We conclude that the more complicated interaction within a periodic pattern, the longer the convergence time is.

We summarize our results about periodic patterns of the recurrent inhibitory loop. The case study illustrates the periodic patterns we theoretically derived in the previous section. Different types of periodic patterns (only $W_{u}$ and $W_{d}$, self-inhibitory, nearest-neighbor, far-range) may coexist in a subinterval of delay, leading to multistability in the recurrent inhibitory loop. The pattern transition serves as a crucial instrument to generate a large class of periodic patterns. The average time of convergence to a periodic pattern plays an important role in determining potential applications of these periodic patterns in neural information transmission and cognition processing.

\section{Comparison with the Model with Alpha Synaptic Function}

The integrate-and-fire model of recurrent inhibitory loops with an alpha synaptic function is given

$$
\left\{\begin{array}{l}
V_{E}^{\prime}(t)=-V_{E}(t)-I_{i n h}+I_{0} \\
V_{I}^{\prime}(t)=-V_{I}(t)+I_{e x c}
\end{array}\right.
$$

A strong enough stimulus $I_{0}$ causes the excitatory neuron $E$ to emit a sequence of spikes at $t_{E}^{(1)}, t_{E}^{(2)}, \ldots$. The firing of the neuron $E$ excites the inhibitory neuron $I$ to fire at $t_{I}^{(1)}, t_{I}^{(2)}, \ldots$. The excitatory postsynaptic current $I_{e x c}$ in (7.1) is initiated by the presynaptic spikes of the excitatory neuron with a time lag $\tau$ and has the form $I_{e x c}=g_{E} \sum_{k} S\left(t-\tau-t_{E}^{(k)}\right)$, where $g_{E}$ is synaptic strength from the excitatory neuron to the inhibitory neuron. In turn, the inhibitory neuron $I$ instantaneously delivers inhibitory postsynaptic potentials (IPSP) to inhibit the firing of the excitatory neuron $E$. The inhibitory synaptic current has the form $I_{i n h}=g_{I} \sum_{k} S\left(t-t_{I}^{(k)}\right)$, where $g_{I}$ is 
synaptic strength from the inhibitory neuron to the excitatory neuron. In this section, we consider the synaptic update function $S(t)$ as an alpha function, given by

$$
S(t)= \begin{cases}\alpha^{2} t e^{-\alpha t} & \text { if } t \geq 0 \\ 0 & \text { otherwise }\end{cases}
$$

We assume that the excitatory postsynaptic current $g_{E} S(t)$ contributed by one spike of the excitatory neuron will cause the inhibitory neuron $I$ to fire and trigger only one spike. We also assume that the inhibitory postsynaptic current $g_{I} S(t)$ contributed by one spike of the inhibitory neuron will cause the potential of the excitatory neuron $E$ to decline.

We take parameter values as $\vartheta=1 \mathrm{mV}, I_{0}=1.5 \mu \mathrm{A}, \alpha=6.0, g_{E}=1.8, g_{I}=1.2, T_{F R}=$ $0.35 \mathrm{~ms}, V_{A}=-0.1187 \mathrm{mV}$. Then

$$
T_{A \vartheta}=\log \left[\frac{I_{0}-V_{A}}{I_{0}-\vartheta}\right]=1.1748 \mathrm{~ms}, \quad T=T_{F R}+T_{A \vartheta}=1.5248 \mathrm{~ms} .
$$

In what follows, we take $\tau^{\prime}=\tau+0.3776$ to discuss the periodic patterns exhibited by the excitatory neuron in the interval $\tau^{\prime} \in[n T, n T+T)$ with a positive integer $n$. Value 0.3776 is only an empirical constant but periodic patterns do not depend on this value.
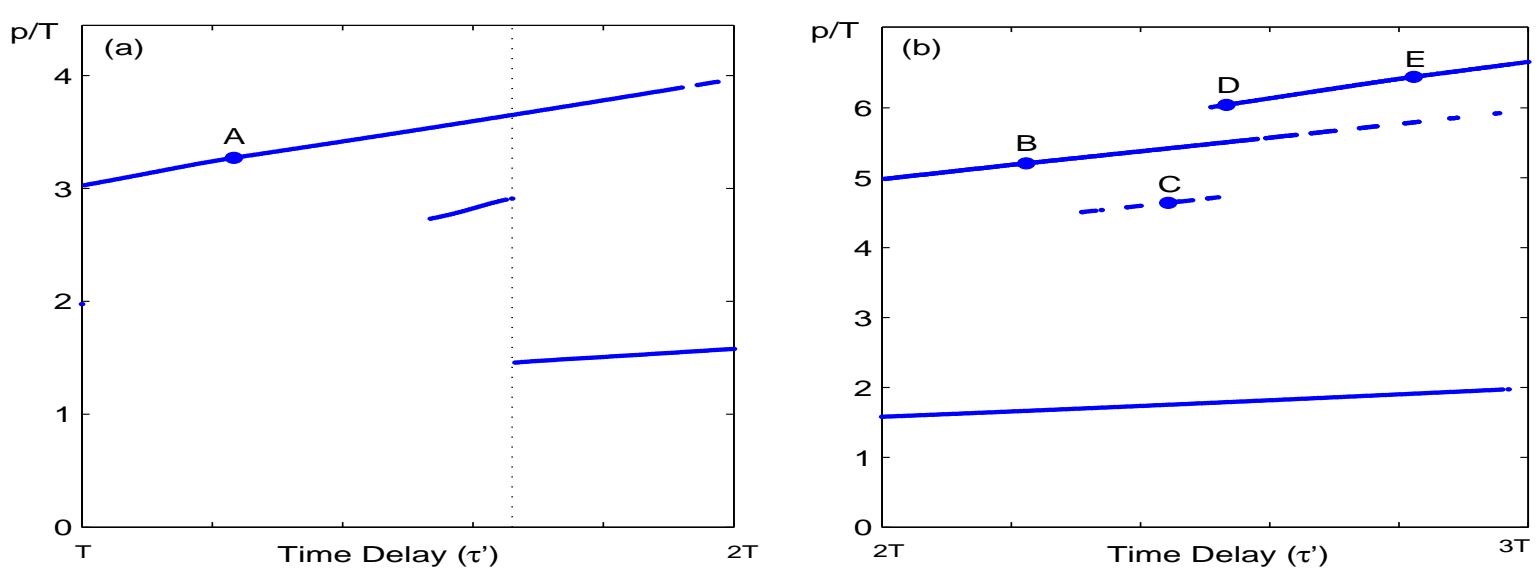

Figure 8: Plot of $p / T$ versus $\tau^{\prime}$ when $\tau^{\prime} \in[T, 2 T)$ (a) and $\tau^{\prime} \in[2 T, 3 T)$ (b). (a) From the bottom to the top, periodic patterns are $\left(W_{u}\right)$ with $\sigma=\left(2 W_{u}\right),\left(W_{u} W_{d}\right),\left(W_{d u} V\right)$ and $\left(W_{u u} V\right)$. The periodic pattern $\left(W_{d u} V\right)$ transits to the periodic pattern $\left(W_{u u} V\right)$ at $A$ and the periodic pattern $\left(W_{u} W_{d}\right)$ transits to the periodic pattern $\left(W_{u}\right)$ with $\sigma=\left(2 W_{u}\right)$ at the dotted line. (b) From the bottom to the top, periodic patterns are $\left(W_{u}\right)$ with $\sigma=\left(2 W_{u}\right),\left(W_{d u} W_{d} V\right),\left(W_{u u} W_{d} V\right),\left(W_{d u u} 2 V\right),\left(W_{u u u} 2 V\right)$, $\left(W_{u u} W_{d} V W_{d}\right),\left(W_{u u} W_{u} V W_{d}\right)$ and $\left(W_{u u} W_{u} V W_{u}\right)$. The periodic pattern $\left(W_{d u} W_{d} V\right)$ transits to the periodic pattern $\left(W_{u u} W_{d} V\right)$ at $C$; the periodic pattern $\left(W_{d u u} 2 V\right)$ transits to the periodic pattern $\left(W_{u u u} 2 V\right)$ at $B$; the periodic pattern $\left(W_{u u} W_{d} V W_{d}\right)$ transits to the periodic pattern $\left(W_{u u} W_{u} V W_{d}\right)$ at $D$ and continues to transit to the periodic pattern $\left(W_{u u} W_{u} V W_{u}\right)$ at $E$. 


\begin{tabular}{c|c|c|c|c}
\hline Pattern & Type & Interval of $\tau^{\prime}$ & $\begin{array}{c}\text { Convergence } \\
\text { Time }\end{array}$ & $\begin{array}{c}\text { Average Conver- } \\
\text { gence Time }\end{array}$ \\
\hline$\left(W_{u}\right)$ & $W_{u}$ & $(1.66 T, 2 T]$ & $20 p \sim 60 p$ & $60 \mathrm{~T}$ \\
\hline$\left(W_{d} W_{u}\right)$ & $W_{u}$ and $W_{d}$ & {$[1.54 T, 1.66 T]$} & $18 p \sim 42 p$ & $85 \mathrm{~T}$ \\
\hline$\left(W_{d u} V\right)$ & self-inhibitory & $(T, 1.23 T]$ & $1 p \sim 2.5 p$ & $6 \mathrm{~T}$ \\
$\left(W_{u u} V\right)$ & self-inhibitory & $(1.23 T, 1.97 T)^{*}$ & $1 p \sim 2.5 p$ & $6 \mathrm{~T}$ \\
\hline
\end{tabular}

Table 4: Periodic patterns on the interval $\tau^{\prime} \in[T, 2 T)$ of the model with the alpha synaptic function. The interval marked by '*' has two subintervals.

\subsection{Periodic Patterns on the Interval $\tau^{\prime} \in[T, 2 T)$}

Comparing Figure 8 (a) with Figure 6 (a), the model of the recurrent inhibitory loop with the alpha synaptic function exhibits the similar periodic patterns as the model with the step feedback function on the interval $\tau^{\prime} \in[T, 2 T)$. On the interval $\tau^{\prime} \in[T, 2 T)$, there exist four periodic patterns, shown in Table 4 and Figure 8 (a): $\left(W_{u}\right)$ with $\sigma=\left(2 W_{u}\right),\left(W_{d} W_{u}\right)$, the self-inhibitory periodic patterns $\left(W_{d u} V\right)$ and $\left(W_{u u} V\right)$. The periodic pattern $\left(W_{d u} V\right)$ transits to the periodic pattern $\left(W_{u u} V\right)$ at $A$ and the periodic pattern $\left(W_{u} W_{d}\right)$ transits to the periodic pattern $\left(W_{u}\right)$ with $\sigma=\left(2 W_{u}\right)$ at the dotted line in Figure $8(\mathrm{a})$. Periodic patterns $\left(W_{u u} V\right)$ and $\left(W_{d} W_{u}\right)$ coexist on the interval $\tau^{\prime} \in[1.54 T, 1.66 T)$ and periodic patterns $\left(W_{u u} V\right)$ and $\left(W_{u}\right)$ coexist on the interval $\tau^{\prime} \in[1.66 T, 1.92 T] \cup[1.945 T, 1.97 T)$. The periodic pattern $\left(W_{u u} V\right)$ exists in two subintervals $(1.23 T, 1.92 T]$ and $[1.945 T, 1.97 T)$. Compared with their average time of convergence, the selfinhibitory periodic patterns $\left(W_{d u} V\right)$ and $\left(W_{u u} V\right)$ are faster to be recognized than the periodic patterns $\left(W_{u}\right)$ and $\left(W_{u} W_{d}\right)$.

\subsection{Periodic Patterns on the Interval $\tau^{\prime} \in[2 T, 3 T)$}

Comparing Figure 8 (b) with Figure 6 (b), the model of recurrent inhibitory loops with the alpha synaptic function exhibits the similar periodic patterns as the model with the step feedback function on the interval $\tau^{\prime} \in[2 T, 3 T)$ : $\left(W_{u}\right)$ with $\sigma=\left(2 W_{u}\right)$, the self-inhibitory periodic patterns $\left(W_{d u u} 2 V\right)$ and $\left(W_{\text {uиu }} 2 V\right)$, and the nearest-neighbor-inhibitory periodic patterns $\left(W_{d u} W_{d} V\right)$, $\left(W_{u u} W_{d} V\right),\left(W_{u u} W_{d} V W_{d}\right),\left(W_{u u} W_{u} V W_{d}\right)$ and $\left(W_{u u} W_{u} V W_{u}\right)$. The periodic pattern $\left(W_{d u} W_{d} V\right)$ transits to the periodic pattern $\left(W_{u u} W_{d} V\right)$ at $C$; the periodic pattern $\left(W_{d u u} 2 V\right)$ transits to the periodic pattern $\left(W_{u u u} 2 V\right)$ at $B$; the periodic pattern $\left(W_{u u} W_{d} V W_{d}\right)$ transits to the periodic pattern $\left(W_{u u} W_{u} V W_{d}\right)$ at $D$ and continues to transit to the periodic pattern $\left(W_{u u} W_{u} V W_{u}\right)$ at $E$ in Figure 8 (b). However, the periodic patterns $\left(W_{d u} W_{d} V\right),\left(W_{u u} W_{d} V\right)$ and $\left(W_{u u u} 2 V\right)$ are separated into several segments in their intervals. Compared with their average time of convergence in Table 5 , the self-inhibitory periodic patterns $\left(W_{\text {du }} 2 \mathrm{~V}\right)$ and $\left(W_{\text {uиu }} 2 \mathrm{~V}\right)$ are faster to be recognized than the nearest-neighbor-inhibitory periodic patterns, and also faster than the periodic patterns composed of only $W_{u}$-oscillations. 


\begin{tabular}{c|c|c|c|c}
\hline Pattern & Type & Interval of $\tau^{\prime}$ & $\begin{array}{c}\text { Convergence } \\
\text { Time }\end{array}$ & $\begin{array}{c}\text { Average Conver- } \\
\text { gence Time }\end{array}$ \\
\hline$\left(W_{u}\right)$ & $W_{u}$ & {$[2 T, 2.97 T]$} & $20 p \sim 60 p$ & $60 \mathrm{~T}$ \\
\hline$\left(W_{d u} W_{d} V\right)$ & nearest-neighbor & {$[2.31 T, 2.44 T]^{*}$} & $2 p \sim 8 p$ & $23 \mathrm{~T}$ \\
$\left(W_{u u} W_{d} V\right)$ & nearest-neighbor & $(2.44 T, 2.52 T]^{*}$ & $2 p \sim 8 p$ & $23 \mathrm{~T}$ \\
\hline$\left(W_{d u u} 2 V\right)$ & self-inhibitory & $(2 T, 2.22 T]$ & $0.5 p \sim 1.5 p$ & $5 \mathrm{~T}$ \\
$\left(W_{u u u} 2 V\right)$ & self-inhibitory & $(2.22 T, 2.95 T]^{*}$ & $0.5 p \sim 1.5 p$ & $5 \mathrm{~T}$ \\
\hline$\left(W_{u u} W_{d} V W_{d}\right)$ & nearest-neighbor & {$[2.51 T, 2.53 T]$} & $14 p \sim 36 p$ & $150 \mathrm{~T}$ \\
$\left(W_{u u} W_{u} V W_{d}\right)$ & nearest-neighbor & $(2.53 T, 2.82 T]$ & $8 p \sim 20 p$ & $87 \mathrm{~T}$ \\
$\left(W_{u u} W_{u} V W_{u}\right)$ & nearest-neighbor & $(2.82 T, 3 T]$ & $7 p \sim 16 p$ & $75 \mathrm{~T}$ \\
\hline
\end{tabular}

Table 5: Periodic patterns on the interval $\tau^{\prime} \in[2 T, 3 T)$, where $p$ is the period of a pattern for any given $\tau^{\prime}$ in the specific interval. The intervals marked by '*' consist of several separated subintervals.

\subsection{Periodic Patterns on the Interval $\tau^{\prime} \in[3 T, 4 T)$}

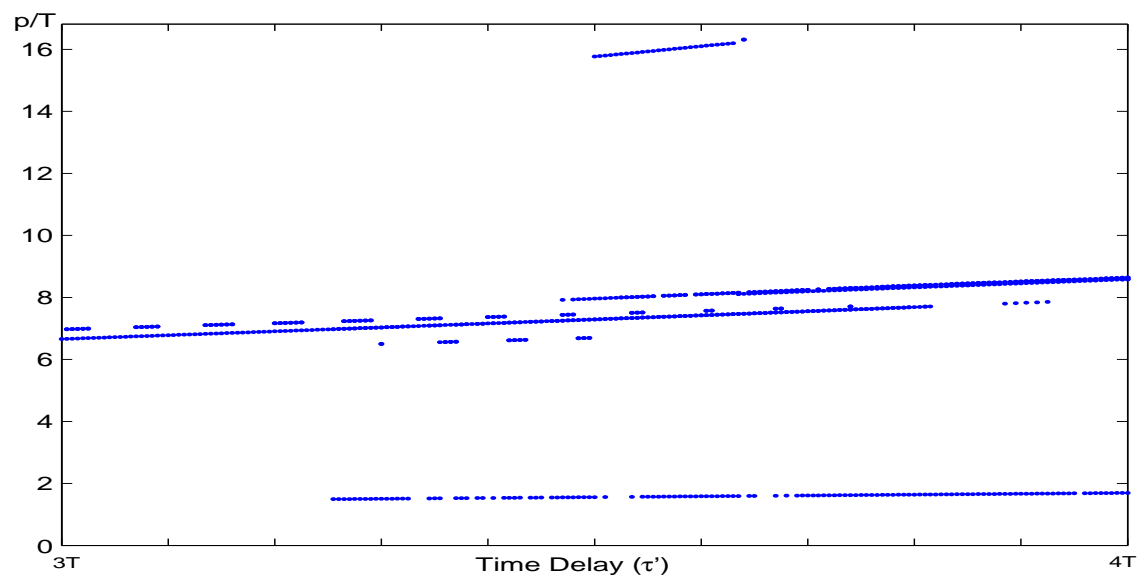

Figure 9: Plot of $p / T$ versus $\tau^{\prime}$ for $\tau^{\prime} \in[3 T, 4 T]$. From the bottom to the top, the periodic patterns are $\left(1 W_{u}\right)$ with $\sigma=\left(3 W_{u}\right), \quad\left(W_{\text {duи }} W_{d} 2 V\right), \quad\left(W_{\text {uиu }} W_{d} 2 V\right)$, $\left(W_{\text {uи }} W_{u} V W_{u}\right),\left(W_{\text {dиии }} 3 V\right),\left(W_{\text {uиии }} 3 V\right),\left(W_{\text {иии }} W_{d} 2 V W_{d}\right),\left(W_{\text {uиu }} W_{u} 2 V W_{d}\right),\left(W_{\text {иии }} W_{u} 2 V W_{u}\right)$, and $\left(W_{u u} W_{d u} V W_{u} V W_{d u} W_{u u} V W_{d} V\right)$. The periodic pattern $\left(W_{d u u} W_{d} 2 V\right)$ transits to the pattern $\left(W_{\text {uиu }} W_{d} 2 V\right)$; the pattern $\left(W_{\text {dииu }} 3 V\right)$ transits to the pattern $\left(W_{\text {uиuи }} 3 V\right)$; the periodic pattern $\left(W_{\text {uиu }} W_{d} 2 V W_{d}\right)$ transits to the pattern $\left(W_{\text {uиu }} W_{u} 2 V W_{d}\right)$ and continues to transit to the pattern $\left(W_{\text {иии }} W_{u} 2 V W_{u}\right)$.

Figure 9 shows periodic pattern on the interval $\tau^{\prime} \in[3 T, 4 T)$ : from the bottom to the top, $\left(W_{u}\right)$ with $\sigma=\left(3 W_{u}\right),\left(W_{\text {duи }} W_{d} 2 V\right),\left(W_{\text {uиu }} W_{d} 2 V\right),\left(W_{\text {uи }} W_{u} V W_{u}\right),\left(W_{\text {duиu }} 3 V\right),\left(W_{\text {uииu }} 3 V\right)$, $\left(W_{\text {uиu }} W_{d} 2 V W_{d}\right),\left(W_{\text {uuu }} W_{u} 2 V W_{d}\right),\left(W_{\text {uиu }} W_{u} 2 V W_{u}\right)$ and $\left(W_{u u} W_{d u} V W_{u} V W_{d u} W_{u u} V W_{d} V\right)$. The 


\begin{tabular}{c|c|c|c|c}
\hline Pattern & Type & Interval of $\tau^{\prime}$ & $\begin{array}{c}\text { Convergence } \\
\text { Time }\end{array}$ & $\begin{array}{c}\text { Average Conver- } \\
\text { gence Time }\end{array}$ \\
\hline$\left(W_{u}\right)$ & $W_{u}$ & {$[3.26 T, 4 T]^{*}$} & $40 p \sim 76 p$ & 93T \\
\hline$\left(W_{d u u} W_{d} 2 V\right)$ & nearest-neighbor & {$[3.30 T, 3.43 T]^{*}$} & $2 p \sim 4 p$ & $20 \mathrm{~T}$ \\
$\left(W_{u u u} W_{d} 2 V\right)$ & nearest-neighbor & {$[3.48 T, 3.50 T)^{*}$} & $0.5 p \sim 3 p$ & $11 \mathrm{~T}$ \\
\hline$\left(W_{u u} W_{u} V W_{u}\right)$ & nearest-neighbor & {$[3 T, 3.93 T]^{*}$} & $9 p \sim 16 p$ & $91 \mathrm{~T}$ \\
\hline$\left(W_{d u u u} 3 V\right)$ & self-inhibitory & $(3 T, 3.22 T]^{*}$ & $0.5 p \sim 1 p$ & $5 \mathrm{~T}$ \\
$\left(W_{\text {uuиu }} 3 V\right)$ & self-inhibitory & $(3.27 T, 3.74 T]^{*}$ & $0.5 p \sim 1 p$ & $5 \mathrm{~T}$ \\
\hline$\left(W_{\text {uuu }} W_{d} 2 V W_{d}\right)^{*}$ & nearest-neighbor & {$[3.47 T, 3.48 T]$} & $16 p \sim 22 p$ & $151 \mathrm{~T}$ \\
$\left(W_{\text {uиu }} W_{u} 2 V W_{d}\right)$ & nearest-neighbor & $(3.48 T, 3.85 T]^{*}$ & $12 p \sim 32 p$ & $180 \mathrm{~T}$ \\
$\left(W_{\text {uиu }} W_{u} 2 V W_{u}\right)$ & nearest-neighbor & $(3.85 T, 4 T]^{*}$ & $8 p \sim 12 p$ & $85 \mathrm{~T}$ \\
\hline$\#$ & far-rang & {$[3.50 T, 3.64 T]$} & $10 p \sim 18 p$ & $225 \mathrm{~T}$ \\
\hline
\end{tabular}

Table 6: Periodic patterns in the interval $\tau^{\prime} \in[3 T, 4 T)$, where $p$ is the period of a pattern for any given $\tau^{\prime}$ in the specific interval. \# represents $\left(W_{u u} W_{d u} V W_{u} V W_{d u} W_{u u} V W_{d} V\right)$. The intervals marked by ' ${ }^{*}$ ' consist of several separated subintervals.

periodic pattern $\left(W_{d u u} W_{d} 2 V\right)$ transits to the pattern $\left(W_{\text {uиu }} W_{d} 2 V\right)$; the pattern $\left(W_{\text {duиu }} 3 V\right)$ transits to the pattern $\left(W_{\text {uuuu }} 3 V\right)$; the periodic pattern $\left(W_{\text {uuu }} W_{d} 2 V W_{d}\right)$ transits to the pattern $\left(W_{u u u} W_{u} 2 V W_{d}\right)$ and continues to transit to the pattern $\left(W_{u u u} W_{u} 2 V W_{u}\right)$. These periodic patterns may be separated into several segments in their corresponding intervals. Compared with their average time of convergence in Table 6 , the self-inhibitory periodic patterns $\left(W_{\text {диии }} 2 V\right)$ and $\left(W_{\text {иии }} 2 V\right)$ are faster to be recognized than the nearest-neighbor-inhibitory periodic patterns, and also faster than the periodic patterns composed of only $W_{u}$-oscillations. The slowest is the far-range-inhibitory periodic pattern $\left(W_{u u} W_{d u} V W_{u} V W_{d u} W_{u u} V W_{d} V\right)$.

\section{Discussion}

In this paper, we have studied the recurrent inhibitory loop modelled by a system of coupled delay differential equations. Our focus is on the capability of such a recurrent loop to generate multiple periodic patterns of the excitatory neuron. Four factors mainly contribute to the coexistence of multiple periodic patterns for a given set of parameters: the extended delay, rich building blocks (four types basic oscillations), a large class of periodic patterns and pattern transition.

Our study shows that the key to generate a large number of coexisting periodic patterns is the interaction of the excitatory and inhibitory neurons, the inhibitory feedback, the incorporation of firing and absolute refractory period in the model of the recurrent loop. First of all, two biological features of individual neurons, the firing and absolute refractory period, make possible three types of basic oscillations of the excitatory neuron: $\left(V, W_{u}\right.$ and $\left.W_{d}\right)$. Secondly, the inhibitory neuron not only inhibits the firing of the excitatory neuron but also extends the neuron's relaxation time, in turn, the prolonged firing duration allows more signals to be delivered, and as a result, multi- 
ple signals create more complicated oscillations $\left(W_{c}\right)$ of the excitatory neuron. Furthermore, in addition to the synaptic delay, the inhibitory neuron, upon receiving an excitatory signal from the excitatory neuron, delivers an inhibitory feedback to the excitatory neuron with an extra delay.

Since the size of the additional delay associated with the specific type of oscillation of the inhibitory neuron is fully determined by the period of the corresponding type of oscillation of the excitatory neuron, the inhibitory signal from the inhibitory neuron can be regarded as a self-feedback of the excitatory neuron with the additional delay. In terms of self-feedbacks, the interaction of these basic oscillations determines the type of periodic patterns: periodic patterns composed of only $W_{u}$-oscillation and $W_{d}$-oscillation, self-inhibitory patterns, nearest-neighbor-inhibitory patterns and far-range-inhibitory patterns. Furthermore, the complicated inhibitory interaction of these basic oscillations plays an important role in eventually stabilizing a trajectory into a periodic solution with one of these predictable periodic patterns. This allows us to link the solution semiflow to symbolic dynamics in which these basic oscillations can be pinned together to form a large class of periodic patterns and make the model exhibit rich dynamics, such as the coexistence of multiple patterns and pattern transition. Different types of periodic patterns may coexist in a subinterval of delay, leading to the occurrence of multistability in the recurrent inhibitory loop. The pattern transition plays a similar role to the standard bifurcation theory in terns of the birth and continuation of multiple periodic patterns.

Although our theoretical analysis is based on the integrate-and-fire model with a step synaptic update function, the same four types of periodic patterns we theoretically derived have been exhibited by our simulation in the more neural model of the recurrent loop with an alpha synaptic update function. The coexistence of multiple periodic patterns and pattern transitions also occur over a wide range of the ratio $\tau^{\prime} / T$ in this realistic model. We defined and numerically calculated the average time of convergence to a periodic pattern to measure the recognizing time of the pattern. Among these periodic patterns, the first three periodic patterns have faster speed to be recognized, hence, they have the potential to be used for neural information transmission and cognition processing in the nervous system. The long recognizing time of far-range-inhibitory patterns prevents them from potential applications in neural signal processing. The more realistic neural model of the recurrent inhibitory loop with the alpha synaptic function presented the same periodic patterns we derived analytically.

\section{References}

[1] C. A. Bares, M. S. Suster, J. Shen, B. L. McNaughton. Multistability of cognitive maps in the hippocampus of old rats. Nature, 388, 272-275 (1997).

[2] A. Beuter, J. G. Milton, C. Labrie, L. Glass. Complex motor dynamics and control in multiloop negative feedback systems. Proc IEEE Systems Man Cybern., 899-902 (1989).

[3] R. M. Borisyuk, A. Kirillov. Bifurcation analysis of a neural network model. Biological Cybernetics, 66 319-325 (1992). 
[4] C. Canavier, D. Baxter, J. Clark, J. Byrne. Multiple modes of activity in a neuron model suggest a novel mechanism for the effects of neuromodulators. J. Neurophysiol., 72, 872-882 (1994).

[5] C. C. Chow, J. A. White, J. Ritt, N. Kopell. Frequency control in synchronized networks of inhibitory neurons. Neural Comput., 5, 407-420 (1998).

[6] D. Cotreras, A. Destexhe, T. J. Sejnowski, M. Steraide. Control of spatiotemporal coherence of a thalamic oscillation by corticothalamic feedback. Science, 274, 771-774 (1996).

[7] G. B. Ermentrout, N. Kopell. Fine structure of neural spiking and synchronization in the presence of conduction delays. Proc. Nat. Acad. Sci., 95, 1259-1264 (1998).

[8] J. Foss, A. Longtin, B. Mensour, J. Milton. Multistability and delayed recurrent loops. Phys. Rev. Lett., 76, 708-711 (1996).

[9] J. Foss, F. Moss, J. Milton. Noise, multistability, and delayed recurrent loops. Phys. Rev. E 55, 4536-4543 (1997).

[10] J. Foss, J. Milton. Multistability in recurrent neural loops arising from delay. J. Neurophysiol., 84(2) 975-985 (2000).

[11] M. J. Gutnick, D. A. Prince. Thalamocortical relay neurons: antidromic invasion of spikes from a cortical epileptogenic focus. Science, 176, 424-426 (1972).

[12] A. C. Guyton. Textbook of medical physiology. Saunders, Toronto, 1976.

[13] J. J. Hopfield. Neural networks and physical systems with emergent collective computational abilities. Proc. Natl. Acad. Sci., 79, 2554-2558 (1982).

[14] J. J. Hopfield. Neurons with grades response have collective computational properties like those of two-state neurons. Proc. Natl. Acad. Sci., 81, 3088-3092 (1984).

[15] N. Kopell, G. B. Ermentrout, M. A. Whittington, R. D. Traub. Gamma rhythms and beta rhythms have different synchronization properties. PNAS, 97, 1867-1872 (2000).

[16] N. Kopell, D. Pervouchine, H. G. Rotstein, T. Netoff, M. Whittington, T. Gloveli. Multiple rhythms and switches in the nervous system. In press.

[17] J. Ma, J. Wu. Multistability in spiking neuron models of delayed recurrent neural loops. Neural Comput., 19, 2124-2148 (2007).

[18] J. Ma, J. Wu. Transition and coexistence of periodic patterns in spiking neuron models of delayed recurrent inhibitory loops. Submitted to SIAM J. Appl. Math..

[19] J. Milller. What is the contribution of axonal conduction delay to temporal structure in brain dynamics? 53-57. In: Oscillatory event-related brain dynamics. C. Pantev, Ed. Plenum, New York, 1994. 
[20] J. Milton. Epilepsy: Multistability in a dynamic disease. In: Self-organized biological dynamics and nonlinear control. J. Walleczek, Ed. Cambridge University Press, Cambridge, 374-386, 2000.

[21] J. Milton. Insights into seizure propagation from axonal conduction times. In: Epilepsy as a dynamic disease. J. Milton, P. Jung, Eds. New York. Springer-Verlag, 15-23 (2002).

[22] M. Morita. Associative memory with non-monotone dynamics. Neural Networks, 6, 115-123 (1993).

[23] M. Proctor, K. Gale. Basal Ganglia and Brainstem Anatomy and Physiology, In: Epilepsy: A comprehensive textbook. J. Engel, T. A. Pedley, Eds. Philadelphia, PA: Lippincott-Raven, 353-368 (1997).

[24] P. A. Schwartzkroin, D. C. McIntyre. Limbic anatomy and physiology. In: Epilepsy: a comprehensive textbook. J. Engel, T. A. Pedley, Eds. Philadelphia, PA: Lippincott-Raven, 323340 (1997).

[25] P. Tiňo, B. G. Horne, C. L. Giles. Attractive periodic sets in discrete-time recurrent networks with emphasis on fixed-point stability and bifurcations in two-neuron networks. Neural Comput., 13, 1379-1414 (2001).

[26] R.D. Traub, R. Miles. Neuronal networks of the hippocampus. Cambridge University Press, New York, 1991. 